\title{
Controlled actuation of therapeutic nanoparticles: an update on recent progress
}

\begin{abstract}
A primary envisioned use for nanoparticles (NPs) in a cellular context is for controlled drug delivery where the full benefit of NP attributes (small size, large drug cargo loading capacity) can improve the pharmacokinetics of the drug cargo. This requires the ability to controllably manipulate the release of the drug cargo from the NP vehicle or 'controlled actuation.' In this review, we highlight new developments in this field from 2013 to 2015. The number and breadth of reports are a testament to the significant advancements made in this field over this time period. We conclude with a perspective of how we envision this field to continue to develop in the years to come.
\end{abstract}

First draft submitted: 14 January 2016; Accepted for publication: 23 March 2016; Published online: 14 April 2016

Keywords: actuation $\bullet$ nanoparticle $\bullet$ therapeutic

The use of nanomaterials for biological applications has grown steadily over the past 15 years and the implementation of bio/nano hybrids (composite materials that incorporate both biologicals and nanomaterials) in cells, tissues and whole organisms continues to expand at a rapid pace. In that time, the role of nanoparticle (NP) materials has transitioned from that of a mere label for cellular imaging [1] to a more active role for making cellular measurements and sensing [2-4]. Much of this progress has been facilitated by our furthered understanding of how NPs can be interfaced with cells and controlled to elicit desired cellular responses [5]. One area that has seen explosive growth is the use of NPs for controlled drug delivery. NPmediated drug delivery (NMDD) seeks to take advantage of the inherent properties of NPs as a means to improve drug circulation time, efficacy and therapeutic index [6-8]. Such properties include:

- NPs' small size for rapid circulation, tissue penetration and clearance

- Large surface area and core volume for significant drug cargo loading
- The ability to be rendered biocompatible and colloidally stable with surface ligands

- Amenability to be bioconjugated with targeting moieties (e.g., peptides, proteins, drugs) for specific cellular homing and uptake

A chief goal of NMDD is the reduced need for repeated, systemic drug dosing regimens by delivering larger, persistent drug doses in the form of a NP construct. Indeed, a PubMed search for the criteria 'nanoparticle + therapeutic' indexed from 2013 to 2015 returns $>10,000$ citations, demonstrating the enormous amount of work occurring in this area. Ultimately, these endeavors will enable the development of theranostic materials; materials that can provide simultaneous disease diagnosis and treatment on a single NP vector.

Integral to success in NMDD is the ability to elicit fine control over the release of the NP-associated drug cargo. In our previous review [9] and editorial [10], we defined this process of controlled cargo release as 'NP actuation' and we further delineated three
Okhil K Nag', Lauren D Field'1, YungChia Chen', Ajmeeta Sangtani', Joyce $C$ Breger $^{1}$ \& James B Delehanty ${ }^{*, 1}$

${ }^{1}$ Center for Bio/Molecular Science \& Engineering, Code 6900, U.S. Naval Research Laboratory, Washington, DC 20375, USA

*Author for correspondence: james.delehanty@nrl.navy.mil

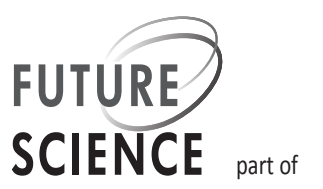


basic modalities of NP actuation: passive; active/ extracellularly triggered; and, active/intracellularly triggered (Figure 1). Passive actuation involves the simple efflux of the drug cargo from the NP surface or from within the NP core. The two active forms of NP actuation utilize either extracellularly applied stimuli (e.g., light [11,12], magnetic field [13]) or intracellularly applied triggers (e.g., $\mathrm{pH}$ [14], redox [15]). While these modalities apply to both 'soft' (e.g., polymers, liposomes) and 'hard' NPs (e.g., metal oxides, semiconductor quantum dots, carbon nanotubes), there are differences in the degree of spatiotemporal control afforded by each. Passive actuation (based on efflux/ desorption) allows the least degree of temporal control and can occur both at the cell's plasma membrane as well as inside the cell. Likewise, active/extracellularly triggered stimuli can be applied to NPs at the plasma membrane or once the NP is internalized. Active/intracellularly triggered actuation occurs via innate cellular triggers inside the cell. Active actuation modalities, since they are directly tied to an applied stimulus, allow tighter temporal control of drug cargo release.

Our goal in this review is to provide an update to our 2013 review [9] and to present new examples of each actuation modality while comparing and contrasting the inherent advantages of the various NP systems/designs. We specifically limit our discussion to those research reports published from 2013 to the present. We also highlight the current state of the use of therapeutic NPs in the clinical setting. Finally, we offer a brief forward-looking perspective on where we expect to see the next significant developments in the therapeutic NP actuation arena as fundamental work progresses.

\section{Controlled actuation of therapeutic NPs}

In this section, we cover in detail recent examples of the various NP actuation modalities. We compare and contrast the levels of control afforded by each modality while highlighting the specific benefits of targeted ligand (e.g., peptide, protein)-mediated delivery. These examples of passive and active actuation are summarized in Tables 1 \& 2, respectively.

\section{Passive NP actuation}

Passive nanoparticle actuation involves the complexation of a cargo (e.g., therapeutic drug, imaging/contrast agent) with an NP carrier followed by the release of the agent by the simplest mechanism known; the passive desorption of the agent from the NP surface or its efflux from within the NP core. Hence, it is often helpful, in the context of passive NP actuation, to think of the NP as either 'hard' or 'soft.' Hard NPs generally refer to those NPs whose surface is the only accessible surface area for cargo adsorption. Examples here include metal NPs such as gold $(\mathrm{Au})$ or silver $(\mathrm{Ag})$ NPs and metal oxides. Soft NPs, on the other hand, are cavernous or otherwise 'core-loadable' and can accommodate cargo within the NP center (e.g., protein nanocages and polymeric NPs such as micelles and liposomes).

Some NPs, in their as-synthesized form, can act directly as the therapeutic agent. For example, AgNPs have been historically used for their innate antimicrobial effects which has led to their use in topical skin applications for burn treatment, in wound dressings and in implanted medical devices to mitigate infection [16-18]. AgNPs can also be used as a toxic agent for the targeted killing of cancerous cells, as was recently shown by the Premanathan group who employed 10 $\mathrm{nm}$ diameter AgNPs to induce apoptosis in human myeloblastic leukemia cells [19]. Additionally, Suliman et al. determined that the time- and dose-dependent cytotoxicity of bare AgNPs was attributable to induced oxidative stress and DNA damage [20]. These types of cytotoxicity results are not universal, however, as was shown by Zhang et al. who analyzed AgNPinduced cytotoxicity in six different mammalian cell lines ranging from epithelial cells to macrophages. Their results showed that differences in innate antioxidant responses and the expression of certain protective proteins (e.g., metallothionein) vary in a cell typedependent manner, resulting in variations in observed AgNP toxicity [21]. Alternatively, particles of a benign nature can be coated with a desired therapeutic agent for passive actuation. Depending on their size [22], capping ligand and route of administration [23], AuNPs can be rather inert and have become a popular delivery vehicle as they can be synthesized over a wide range of sizes and can be combined with small molecule drugs to increase the potency of the therapeutic cargo. One widely studied drug is doxorubicin (DOX), the anthracycline antitumor antibiotic commonly used in chemotherapy. Numerous groups have studied the efficacy and production of DOX-coated AuNPs, from the determination of a self-assembly method using heavy metal binding proteins (HMBPs) [24] to the synthesis of magnetic AuNPs for magnetically targeted drug delivery (vide infra) [25]. In the former case, recombinantly expressed HMBPs phytochelatin and metallothionein were used to reduce gold ions to form AuNP-HMBP assemblies which elicited minimal toxicity when incubated with HeLa cells [24]. Passive adsorption of DOX onto the AuNP-HMBP surface improved the $\mathrm{IC}_{50}$ of the drug by approximately $30 \%$ compared with free DOX (Figure 2A).

In contrast to relying on nonspecific endocytosis for the cellular internalization of the therapeutic NPs, 


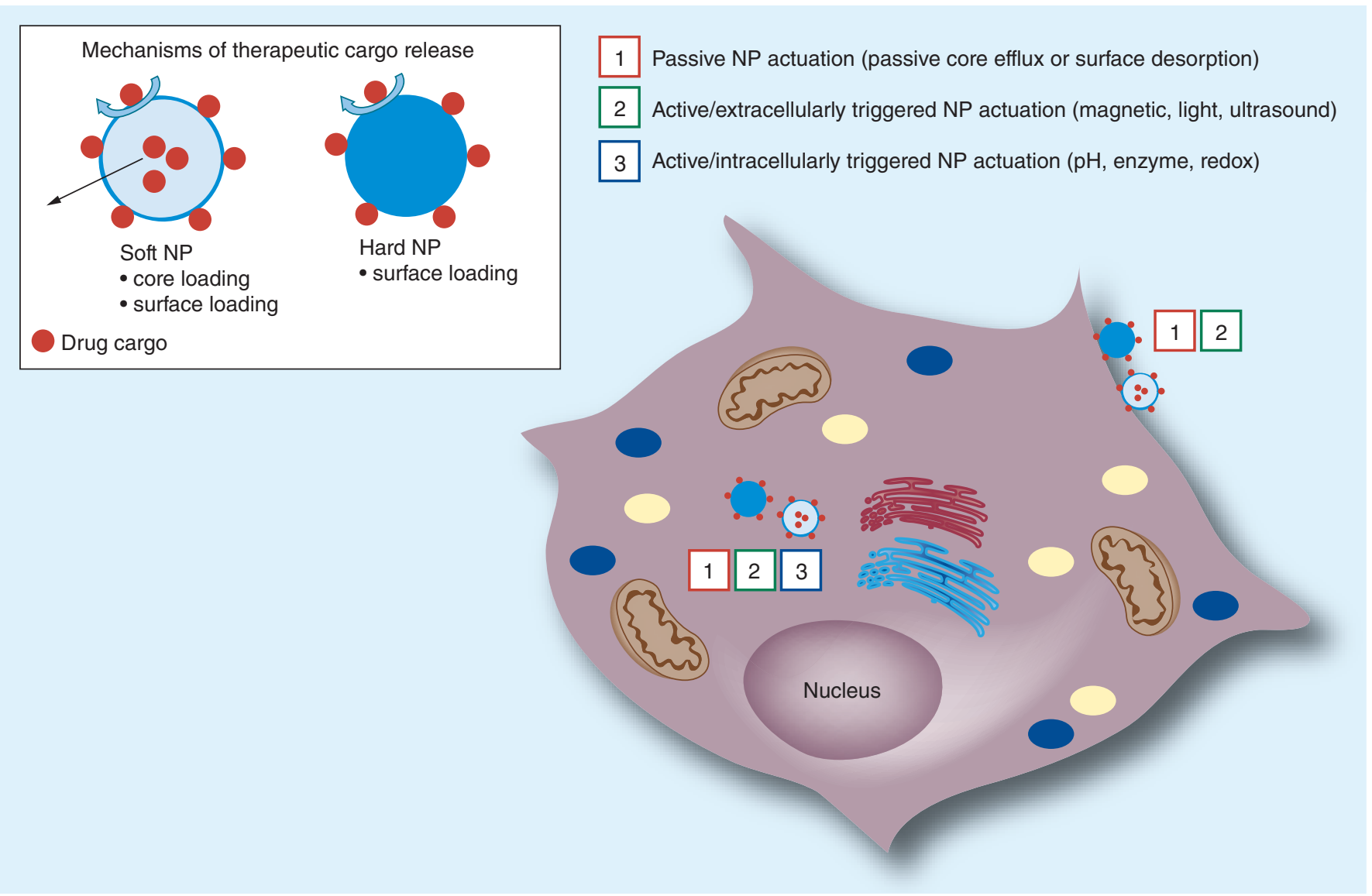

Figure 1. Modes of therapeutic nanoparticle actuation. NP-associated cargos can be actuated or released by three general mechanisms: passive, active/extracellularly triggered or active/intracellularly triggered. (Inset) NP drug cargo release mechanisms. Drug cargos are released from soft and hard NPs in various ways. Soft NPs (e.g., liposomes, micelles) can be loaded in the NP core or on the surface. Hard NPs (e.g., AuNPs, metal oxides, quantum dots) are primarily loaded on their surface. Drug cargo release is indicated with arrows. (Main) Spatiotemporal control of NP actuation. Both soft and hard NPs are subject to actuation by all three modalities. The primary difference is the location of the triggering mechanism as indicated with the numbered boxes. NP: Nanoparticle.

more specific targeting/uptake strategies using specific ligands are employed to localize the NP-drug conjugate to the desired location. Here, we highlight several that have been used in the context of hard particles such as AuNPs and various metal oxide NPs. Afifi et al. functionalized AuNPs with the small molecule inhibitor (XAV939) of the tankyrase enzyme, a poly-ADP-ribosyltransferase involved in the Wnt signaling pathway, to achieve $>100$-fold increase in the inhibitor's efficacy in a manner that tracked with diameter of the AuNP [26]. Iron oxide nanoparticles (IONPs) have also been conjugated to targeting agents, including the antiHER2/neu peptide to target paclitaxel-loaded IONPs to HER2/neu-positive breast cancer cells [27] or the addition of chlorotoxin to target to IONPs to human glioblastoma cells [28]. Both of these systems utilize the passive release of the loaded paclitaxel cargo while specifically targeting the NP through the use of other agents. This multifunctionality is a critical aspect of NP-based drug delivery and Kossatz et al. took this to the next level by conjugating DOX-loaded IONPs with Nucant multivalent nucleolin-targeting pseudopeptide (N6L) [29]. The nucleolin-receptor complex is overexpressed selectively at the cell surface of tumor cells and the N6L peptide mediated efficient NP uptake. This ensemble drug construct could be preferentially localized to cells with an applied magnetic field, induced to be internalized by N6L peptide and then locally heated for combined drug and hyperthermic cell killing. Once demonstrated in MDA-MB-231 breast cancer cells, hyperthermic treatment showed significant improvement in halting tumor progression (up to $40 \%)$ upon intratumoral injection in athymic nude mice (Figure B). Similarly, Elbialy et al. synthesized approximately $22 \mathrm{~nm}$ diameter AuNPs whose core was loaded with AgNPs and surface was covalently conjugated to DOX to drive magnetically assisted enhanced targeting to the tumor site in a model of Ehrlich carcinoma [25]. Semiconductor nanocrystals, or quantum dots (QDs), have also proven to be a very robust 


\begin{tabular}{|c|c|c|c|c|}
\hline $\begin{array}{l}\text { Actuation } \\
\text { method }\end{array}$ & Nanoparticle & Cargo/action & Cell/tissue (purpose) & Ref. \\
\hline \multirow[t]{22}{*}{ Passive } & AgNP & Inherent NP toxicity & Human myeloblastic leukemia cells (apoptosis) & [19] \\
\hline & AgNP & Inherent NP toxicity & Human lung epithelial cells (oxidative stress/DNA damage) & [20] \\
\hline & Au-HMBPs & DOX & HeLa (chemotherapeutic) & [24] \\
\hline & AuNP & $\begin{array}{l}\text { XAV939, tankyrase } \\
\text { inhibitor }\end{array}$ & Human oral squamous cell (chemotherapeutic) & [26] \\
\hline & IONPS & Paclitaxel & Breast cancer cells (chemotherapeutic) & [27] \\
\hline & IONPS & Paclitaxel & Human glioblastoma cells (chemotherapeutic) & [28] \\
\hline & IONPs & DOX & Breast cancer cells (chemotherapeutic) & [29] \\
\hline & AuNP & DOX & Ehrlich carcinoma (chemotherapeutic) & [25] \\
\hline & CdTe QDs & DOX & HeLa (chemotherapeutic) & [30] \\
\hline & Protein nanocages & $\begin{array}{l}\text { OSU03012, } \\
\text { Celecoxib derivative }\end{array}$ & Pancreatic cancer cells (chemotherapeutic) & [32] \\
\hline & Protein nanocages & DOX & HeLa (chemotherapeutic) & [33] \\
\hline & Dendrimers & $\begin{array}{l}\text { Triamcinolone } \\
\text { acetonide }\end{array}$ & $\begin{array}{l}\text { Human retinal pigment epithelium cells (macular } \\
\text { degeneration) }\end{array}$ & [34] \\
\hline & Copolymer micelle NP & Curcumin/oxoplatin & Human ovarian cancer cells (chemotherapeutic) & [35] \\
\hline & Liposomes & Dexamethasone & $\begin{array}{l}\text { Prostate cancer bone metastases in vivo } \\
\text { (chemotherapeutic) }\end{array}$ & [36] \\
\hline & Liposomes & Ruthenium nitrosyl & Hepatocarcinoma cells (inducing apoptosis) & [37] \\
\hline & Liposomes & Methotrexate & CD40L + cells (autoimmune diseases) & [38] \\
\hline & Liposomes & DOX & HUVECs/U87 cells and glioma in vivo (chemotherapeutic) & [39] \\
\hline & Immunoliposomes & Antibodies & Malaria parasite (Antimalarial therapeutic) & [41] \\
\hline & Liquid crystal NPs (LCNPs) & DOX & HEK 293T/17 (chemotherapeutic) & [42] \\
\hline & $\begin{array}{l}\text { Porous silicon } \\
\text { nanoparticle }\end{array}$ & $\begin{array}{l}\text { Sorafenib/ } \\
\text { Methotrexate }\end{array}$ & U87 MG cancer cells (chemotherapeutic) & [43] \\
\hline & $\mathrm{Au}$ in BSA NPs & DOX & HeLa (chemotherapeutic) & [44] \\
\hline & $\begin{array}{l}\text { Graphene QD-human } \\
\text { serum albumin NPs }\end{array}$ & Gemcitabine & Pancreatic cancer (chemotherapeutic) & [45] \\
\hline
\end{tabular}

drug delivery NP platform for passive delivery as their extreme brightness and photostability enables facile tracking of appended cargos. Chen et al. used glutathione (GSH)-coated CdTe QDs that were conjugated to DOX through a condensation reaction and further decorated with folic acid (FA)-terminated poly (ethylene glycol) (PEG) to specifically target HeLa cells [30]. The PEG coating availed minimal nonspecific binding while the FA moiety facilitated localization of the particles and subsequent increased intracellular accumulation of the QD-drug conjugates (Figure 2C). The FA moiety was also used by the Huang group to functionalize DOX-loaded graphene QDs. The change in particle composition allowed for them to capitalize on the properties of graphene to produce particles with very high loading capabilities [31]. These variations on hard particle carriers shows the wide range of available options and the methods through which passive actuation can provide targeted drug delivery.

In contrast to hard NP materials, soft NPs incorporate the therapeutic cargo within the cavernous or porous core and simple efflux drives drug release. The Hashizume group, for example, has developed protein nanocages based on heat shock proteins fused to neuropilin-1 binding peptide (RGD). Flexible linker spacers based on the tripeptide repeat, GGS, were used to controllably separate the RGD motif from the protein nanocage scaffold and the efficiency of uptake by pancreatic cancer cells tracked with increasing linker length [32]. Gentle heating was used to introduce the 

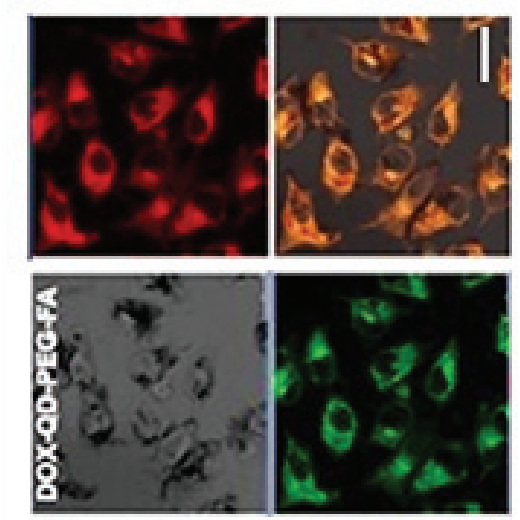

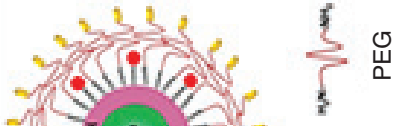
No/now

(0)
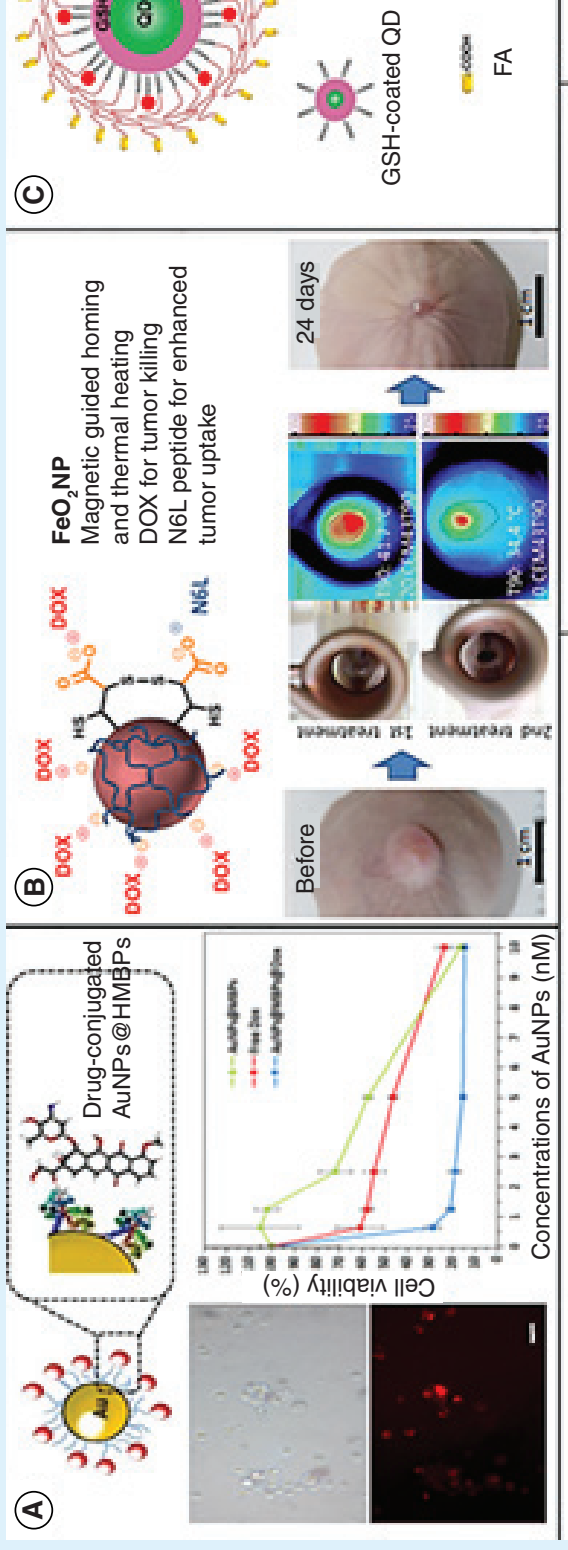

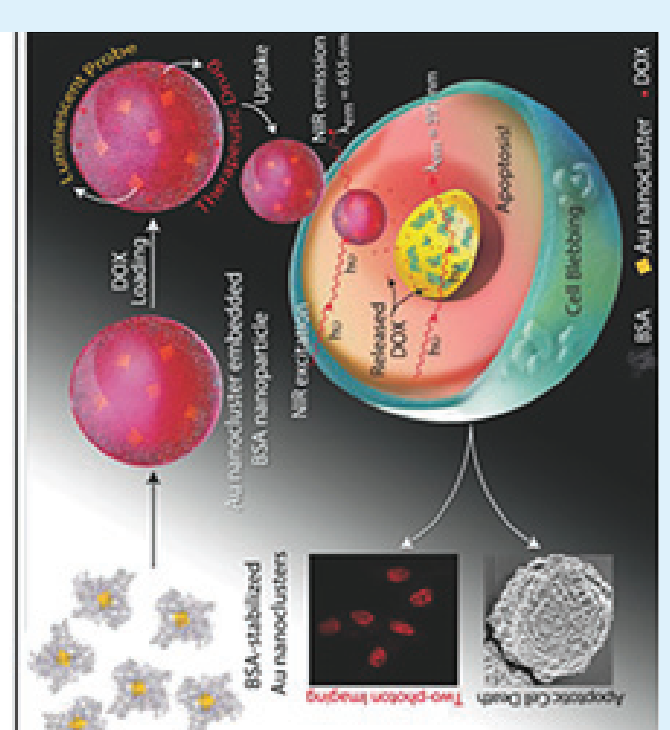

(4)
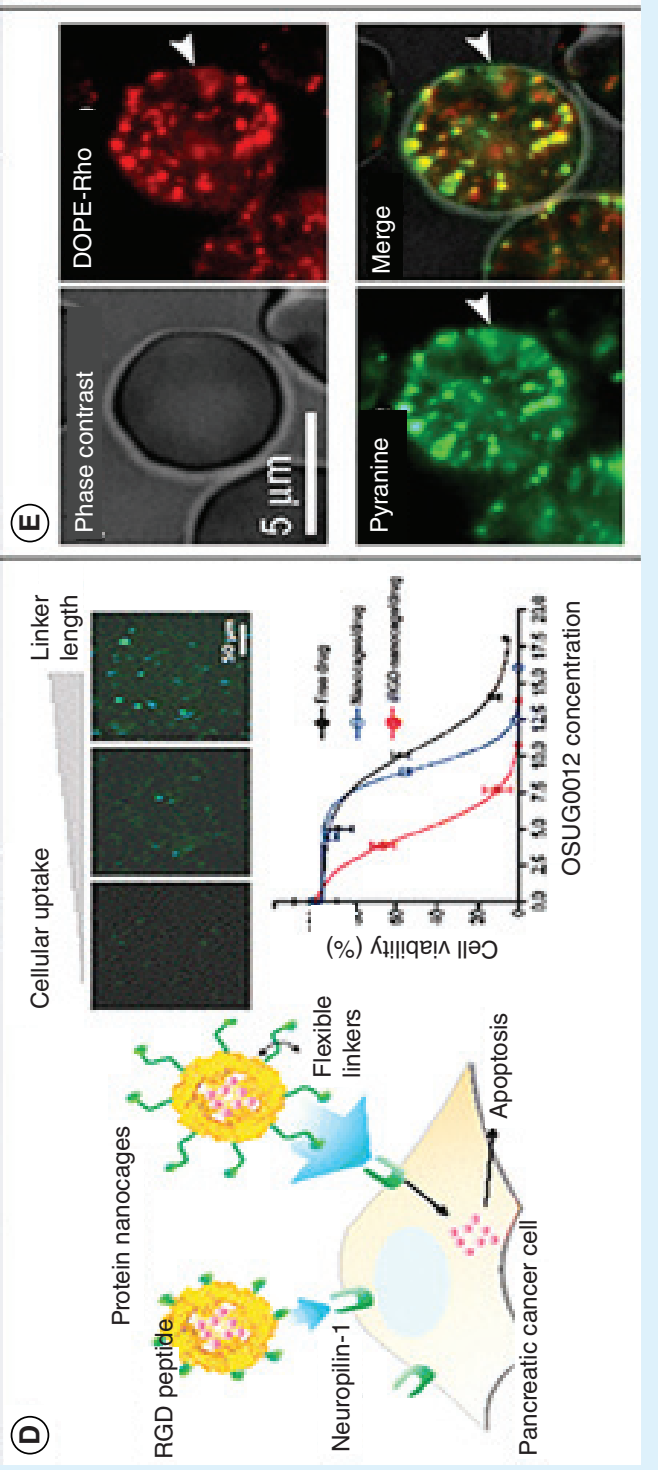

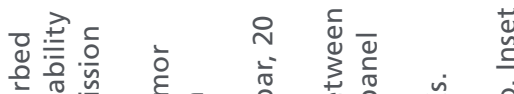

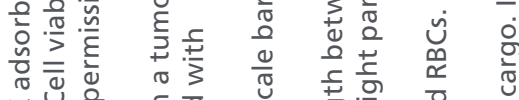
ํ.

娄

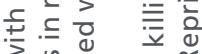

ลิำ

范

主亡

(ㄱ)

는은

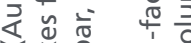

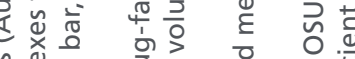

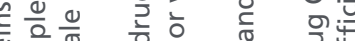

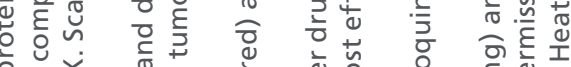

응

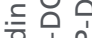

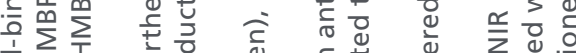

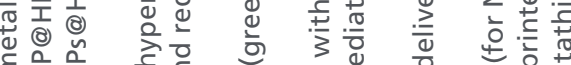

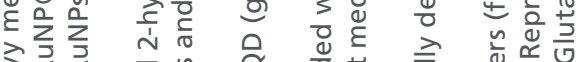

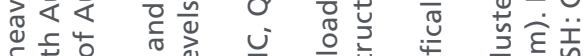

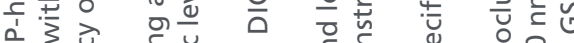

उठ워

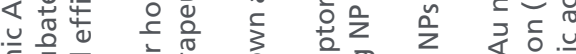

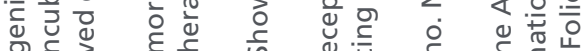

으을

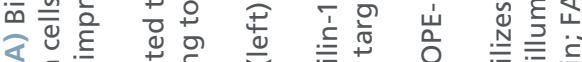

$\leq \pi$

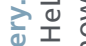

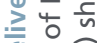

क्ष

올 율

을

ᄃ

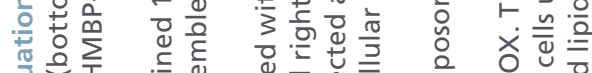

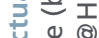

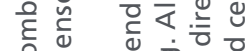

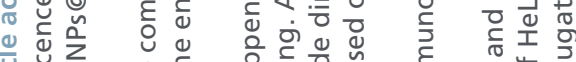

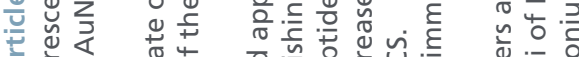

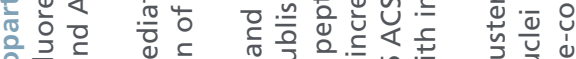

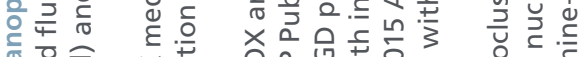

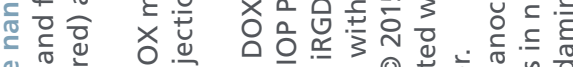

ग

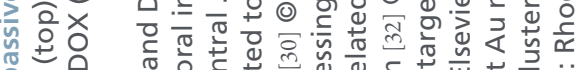

늠 0

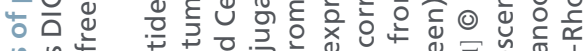

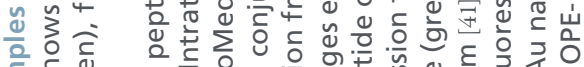

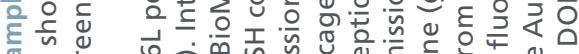

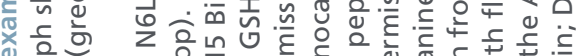

ब

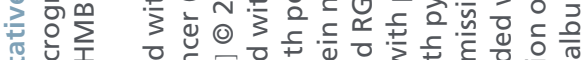

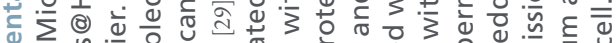

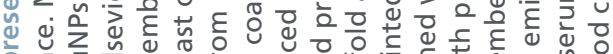

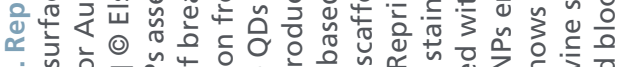
N o

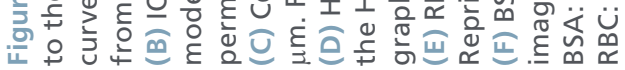


Table 2. Select examples of active actuation modalities for various nanoparticle materials.

\begin{tabular}{|c|c|c|c|c|c|}
\hline Actuation method & $\begin{array}{l}\text { Trigger/ } \\
\text { stimulus }\end{array}$ & Nanoparticle & Cargo/action & Cell/tissue (purpose) & Ref. \\
\hline \multirow[t]{18}{*}{$\begin{array}{l}\text { Active/extracellularly } \\
\text { triggered }\end{array}$} & \multirow[t]{4}{*}{ Magnetic } & $\mathrm{Zn}_{0.4} \mathrm{Fe}_{2.6} \mathrm{O}_{6}$ & DOX & $\begin{array}{l}\text { Mouse tumor xenograft } \\
\text { (chemotherapeutic) }\end{array}$ & [13] \\
\hline & & $\mathrm{Fe}_{3} \mathrm{O}_{4}$-polymer & DOX & $\begin{array}{l}\text { Mouse tumor xenograft } \\
\text { (chemotherapeutic) }\end{array}$ & [48] \\
\hline & & IONPS & Mitoxantrone & $\begin{array}{l}\text { Rabbit tumor xenograft } \\
\text { (chemotherapeutic) }\end{array}$ & [49] \\
\hline & & Liposome-IONPs & $\begin{array}{l}\text { Photosensitizer } \\
\text { m-THPC }\end{array}$ & $\begin{array}{l}\text { Mouse tumor xenograft } \\
\text { (photodynamic therapy) }\end{array}$ & [50] \\
\hline & \multirow[t]{7}{*}{ Light } & Micelles & Camptothecin & ex vivo (chemotherapeutic) & [11] \\
\hline & & Polymer & Nintedanib & $\begin{array}{l}\text { Retinal vasculature (inhibition of } \\
\text { angiogenesis) }\end{array}$ & [12] \\
\hline & & Chitosan-CNT & DOX & HeLa (chemotherapeutic) & [51] \\
\hline & & $\begin{array}{l}\text { Au-cellulose } \\
\text { matrix }\end{array}$ & Aspart & Rat model of hyperglycemia & [52] \\
\hline & & AuNP & DOX & A549 cells (chemotherapeutic) & [53] \\
\hline & & $\mathrm{MoS}_{2}$ nanosheets & DOX & $\begin{array}{l}\text { Tumor in mouse (chemo and } \\
\text { photothermal therapy) }\end{array}$ & [54] \\
\hline & & $\mathrm{CdTe} / \mathrm{CdSe} \mathrm{QD}$ & $\begin{array}{l}\text { Modulate membrane } \\
\text { potential }\end{array}$ & LnCap (prostate cancer) & [55] \\
\hline & \multirow[t]{7}{*}{ Ultrasound } & PLGA & Insulin & Mouse model of hyperglycemia & [60] \\
\hline & & PMAA & DOX & $\begin{array}{l}\text { HeLa cells and tumor } \\
\text { (chemotherapeutic) }\end{array}$ & [61] \\
\hline & & Hydrogel & Mitoxantrone & $\begin{array}{l}\text { Mouse tumor xenograft } \\
\text { (chemotherapeutic) }\end{array}$ & [62] \\
\hline & & Liposomes & Calcein and DOX & $\begin{array}{l}\text { MDA-MB-231 breast cancer cells } \\
\text { (chemotherapeutic) }\end{array}$ & [63] \\
\hline & & Cerasome & DOX & $\begin{array}{l}\text { Mouse adenocarcinoma } \\
\text { (chemotherapeutic) }\end{array}$ & [64] \\
\hline & & Polymer & plasmid DNA & $\begin{array}{l}\text { HepG2 cells (plasmid DNA/dene } \\
\text { delivery) }\end{array}$ & [65] \\
\hline & & PEG-PLGA & microRNA-122 & $\begin{array}{l}\text { Human colon cancer xenografts } \\
\text { (chemotherapeutic) }\end{array}$ & [66] \\
\hline \multirow[t]{7}{*}{$\begin{array}{l}\text { Active/intracellularly } \\
\text { triggered }\end{array}$} & \multirow[t]{4}{*}{$\mathrm{pH}$} & Polymer & DOX & $\begin{array}{l}\text { Mouse tumor xenograft } \\
\text { (chemotherapeutic) }\end{array}$ & [14] \\
\hline & & SMP & DOX & HepG2 cells (chemotherapeutic) & [71] \\
\hline & & AuNP & Manganese ion & $\begin{array}{l}\text { PC12 pheochromacytoma cells } \\
\text { (necrosis and apoptosis) }\end{array}$ & [72] \\
\hline & & IONPs & DOX & $\begin{array}{l}\text { Mouse tumor xenograft } \\
\text { (chemotherapeutic) }\end{array}$ & [73] \\
\hline & \multirow[t]{3}{*}{ Enzyme } & MSNs-peptide & DOX & $\begin{array}{l}\text { HepG2 cells and tumor in mice } \\
\text { (chemotherapeutic) }\end{array}$ & [74] \\
\hline & & Au-'corked'-CNT & Paclitaxel & $\begin{array}{l}\text { Myeloid-derived suppressor cells } \\
\text { (chemotherapeutic) }\end{array}$ & [75] \\
\hline & & $\begin{array}{l}\text { Sulfato- } \beta-C D / \\
\text { protamin }\end{array}$ & DOX & $\begin{array}{l}\text { Hepatic cancer cells } \\
\text { (chemotherapeutic) }\end{array}$ & [77] \\
\hline
\end{tabular}




\section{Table 2. Select examples of active actuation modalities for various nanoparticle materials (cont.).}

\begin{tabular}{|c|c|c|c|c|c|}
\hline \multirow[t]{6}{*}{ Actuation method } & $\begin{array}{l}\text { Trigger/ } \\
\text { stimulus }\end{array}$ & Nanoparticle & Cargo/action & Cell/tissue (purpose) & Ref. \\
\hline & & $\begin{array}{l}\text { Polystyrene- } \\
\text { protine }\end{array}$ & DOX & $\begin{array}{l}\text { Human umbilical vein endothelial cells } \\
\text { (chemotherapeutic) }\end{array}$ & {$[78]$} \\
\hline & Redox & $\begin{array}{l}\text { Mesoporous silica } \\
\text { NP }\end{array}$ & Methotrexate & $\begin{array}{l}\text { U87 MG cancer cells } \\
\text { (chemotherapeutic) }\end{array}$ & [15] \\
\hline & & $\begin{array}{l}\text { Mesoporous silica } \\
\text { NP }\end{array}$ & 6-mercaptopurine & ex vivo & [80] \\
\hline & & $\begin{array}{l}\text { Thiolated gelatin } \\
\text { NPs }\end{array}$ & Gemcitabine & $\begin{array}{l}\text { Mouse pancreatic tumor xenograft } \\
\text { (chemotherapeutic) }\end{array}$ & [82] \\
\hline & & Cerium oxide NPs & Camptothecin & BxPC-3 cells (chemotherapeutic) & [83] \\
\hline
\end{tabular}

moderately hydrophobic anticancer drug, OSU03012, to the NP core and the drug-loaded NPs induced caspase-dependent apoptosis (Figure 2D). A similar protein nanocage platform was reported by the Prosperi group who took advantage of the overexpression of ferritin receptors on cancer cells to deliver apoferritin nanocages loaded with DOX. The ferritin-DOX complexes were internalized and accumulated in the nucleus faster than free DOX, leading to enhanced DOX-induced cell killing [33].

Polymeric NP structures, such as dendrimers and micelles/liposomes continue to be useful delivery platforms for therapeutics as these structures can be loaded with large amounts of cargo payloads. Kambhampati et al. used poly (amidoamine) dendrimers to deliver triamcinolone acetonide (TA) to microglial and human retinal pigment epithelium cells with approximately 100 -fold improved efficacy compared with free TA [34]. This work holds exciting promise for the in vivo treatment of vision-related diseases including diabetic retinopathy and macular degeneration. The Stenzel lab produced a specific tri-block copolymer micelle NP system based on biodegradable PEG with two different compartments; one for the drug curcumin and another for the conjugation of platinum drugs (oxoplatin) [35]. These micelles were shown to have a combinatorial effect when administered to a human ovarian cancer cell line and were even more effective than delivery of a mixture of nanoparticles carrying the drugs separately. One key feature of micelles is their hydrophobic core which provides a suitable environment for poorly water-soluble drugs (e.g., curcumin).

Several groups have used liposomes to carry various cargos for delivery, including dexamethasone for targeting prostate cancer bone metastases [36] and ruthenium nitrosyl to induce apoptotic induction in hepatocarcinoma cells [37]. Functionalization of liposomes with peptides and other biologicals continues to be a highly efficient method of targeting the passive release of the cargo at the desired site of action. Ding et al. used liposomes conjugated to a CD40-specific peptide ligand designed from molecular docking studies to deliver methotrexate (MTX) to CD40 ${ }^{+}$cells for treating autoimmune diseases [38]. Other groups have used peptides to facilitate NP transcytosis across the bloodbrain barrier [39], and to specifically target myocardial infarctions with drug loaded liposomes [40]. More recently, Moles et al. delivered antimalarial drugs such as chloroquine using immunoliposomes coated with antibodies specific to glycophorin A, which is present on red blood cells infected with early intraerythrocytic stages of the malaria parasite Plasmodium falciparum (Figure 2E) [41]. In a similar vain to liposomes, our laboratory has developed liquid crystal NPs (LCNPs) for the combined spatiotemporal two-color tracking of the endocytic pathway and enhanced drug delivery [42]. Here, transferrin conjugated to the LCNP surface drove the efficient endocytosis of the NP materials and the high photostability of the embedded pyrelene fluorophore afforded photostable imaging not achievable with AlexaFluor-transferrin conjugates. Further, these materials could be loaded post synthesis with DOX and delivered to HEK 293T/17 cells to realize a approximately 40 -fold increase in DOX efficacy compared with free DOX.

As fundamental research into passive NP actuation has progressed, so too has the design of more intricate NP constructs aimed at the simultaneous passive actuation of multiple drug cargos. The combinatorial delivery of therapeutic drugs is a common theme in chemotherapy. Wang et al. designed a porous silicon nanoparticle system that could successfully deliver two agents: the hydrophobic drug sorafenib (loaded within the NP core) and MTX (conjugated to the NP surface) to increase cellular uptake and sustained release of the two drugs [43]. Over a $96 \mathrm{~h}$ experimental window, the 
MTX-silicon NPs achieved similar inhibition of cell proliferation in U87 MG cancer cells overexpressing folate receptors (FR) and a higher effect in low FRexpressing EA.hy926 cells compared with free MTX.

In an analogous fashion, other groups have incorporated multiple NP species into a single vehicle to take advantage of the unique features of disparate nanomaterials in a single vehicle platform. Khandelia et al. embedded near infrared-emitting Au nanoclusters into bovine serum albumin (BSA) NPs which were then loaded with DOX to yield a theranostic imaging/drug delivery composite that emits in the $650-900 \mathrm{~nm}$ biological imaging window (Figure 2F) [44]. The BSA NPs acted as a stabilizing host for both the Au nanocluster imaging agent as well as the DOX drug cargo. In HeLa cells, the albumin form of the DOX afforded improved solubility of the drug although it did not significantly improve DOX efficacy compared with free DOX. The authors attributed this to the strong retention of the amphiphilic DOX by the albumin NPs; a feature that could prove advantageous for sustained release in vivo. In a similar fashion, the Sarkar group employed human serum albumin NPs that were functionalized with hyaluronic acid (HA) and conjugated to graphene quantum dots [45]. These NPs were further loaded with the antimetabolite chemotherapeutic drug, gemcitabine, for combined imaging and apoptosis induction in pancreatic cancer cells. The HA moiety directed the NPs to pancreatic cancer cells overexpressing CD-44, a known HA receptor. Overall, these various passive NP actuation modalities demonstrate the wide range of available NP materials that can be imbued with various therapeutic drug cargos for the sustained release of drug cargo, whether from a soft or hard NP platform.

\section{Active NP actuation}

Active NP actuation refers to the application of an external stimulus to the NP to drive the release of an NP-associated cargo (e.g., thermal-induced release of liposome-embedded cargo) or to elicit a physicochemical reaction/response from the NP material itself (e.g., light-induced resonance heating of AuNPs). Active actuation can be further classified based on exactly from where the activating stimulus emanates. In the simplest terms, active actuating stimuli can be located either extracellularly or they can be derived from intracellular cues. These stimuli can be either associated with an inherent cellular process or state (e.g., enzymatic activity, redox, or $\mathrm{pH}$ ) or they can be derived from completely noncellular sources (e.g., laser, magnetic or radiofrequency field, mechanical stress). For purposes of clarity, we divide our discussion of active actuation stimuli into active/extracellular and active/intracellular stimuli.
Throughout this section, it is important to appreciate the key fundamental difference between passive and active NP actuation: spatiotemporal control. Active actuation involves the use of a triggering stimuli to effectively 'do work' on the NP and its associated cargo. As a result, it affords a degree of spatial and temporal control over the NP actuation process that is typically not attainable in passive NP actuation schemes. Accordingly, actively actuated therapeutic NP systems potentially offer great promise for enhanced, ondemand release with fine control over dosing regimens.

\section{Active/extracellularly triggered NP actuation Magnetically triggered actuation}

Magnetic field-assisted NP actuation has become one of the most popular active NP triggering modalities. Here, an externally applied magnetic field is used to modulate the properties of the NP scaffold to affect the release of the NP-associated therapeutic or imaging agent. The use of metal oxides as the active scaffold in this capacity has allowed them to be used as contrast agents for magnetic resonance imaging (MRI), hyperthermic agents in thermal ablation therapy and magnetic vectors for targeted, site-specific delivery. Superparamagnetic iron oxides such as magnetite $\left(\mathrm{Fe}_{3} \mathrm{O}_{4}\right)$ and maghemite $\left(\mathrm{Fe}_{2} \mathrm{O}_{3}\right)$ remain among the most widely employed magnetic NPs (MNPs) due to their biodegradability and often low toxicity, which has been shown in many instances to be cell type-dependent $[46,47]$. Typically, the MNPs are coated with watersoluble polymeric materials such as PEG, dextran, polyethyeneimine (PEI), citric acid or polyvinyl alcohol to impart colloidal stability and stealth. A number of recently reported MNP systems are described below.

Lee et al. developed a DOX-loaded supramolecular, multicomponent MNPs (DOX-SMNPs) based on the self-assembly between the polycyclic hydrocarbon adamantane (Ad) and $\beta$-cyclodextrin (CD) [13]. PEI conjugated to display multiple copies of $\mathrm{CD}$ were selfassembled with $\mathrm{Zn}_{0.4} \mathrm{Fe}_{2.6} \mathrm{O}_{6}$ MNPs, polyamidoamine, PEG (each species bioconjugated to the cognate Ad ligand) and DOX (Figure 3A). The resultant NP size was controlled by the ratiometric amounts of reactants included during assembly. Upon application of an alternating magnetic field (AMF), the embedded MNPs served as a built-in heat transformer to trigger the release of DOX from the core of the magnetothermally responsive SMNP vector. In a mouse tumor xenograft model, intravenous injection of the $70 \mathrm{~nm}$ DOX-loaded SMNPs allowed the usage of 1/1000th of the drug dosage compared with existing protocols. The Ishimura group prepared MNPs comprised of $\mathrm{Fe}_{3} \mathrm{O}_{4}$ NPs and DOX encapsulated within a temperature-sensitive carboxylic polypyrole polymer (glass- 


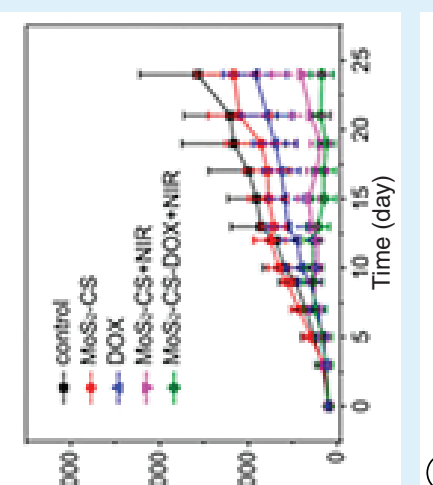

这

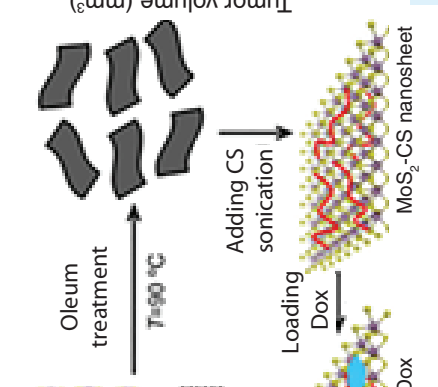

(-)

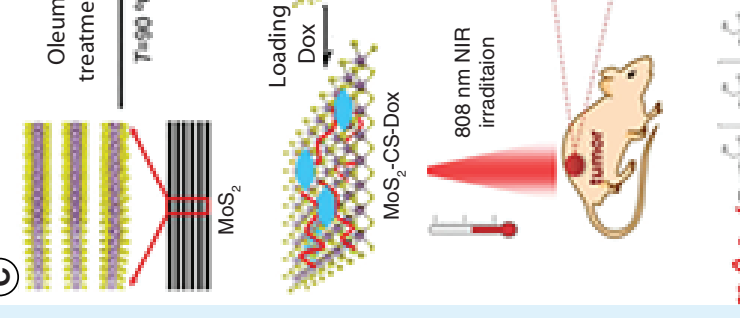

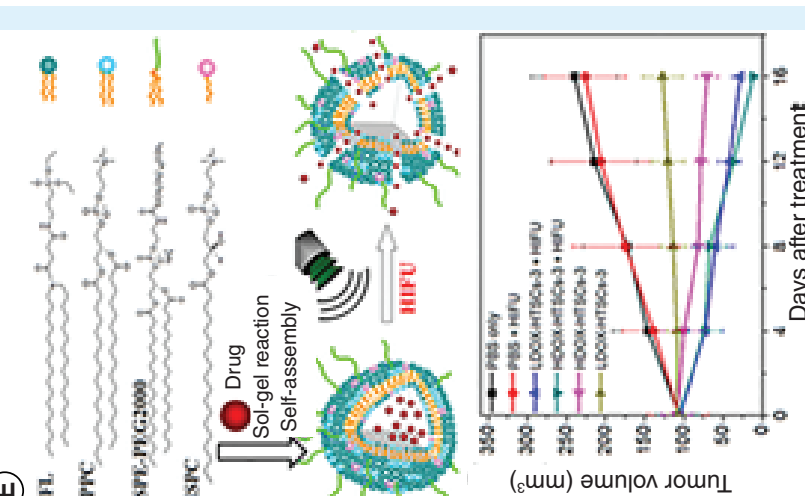

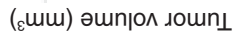
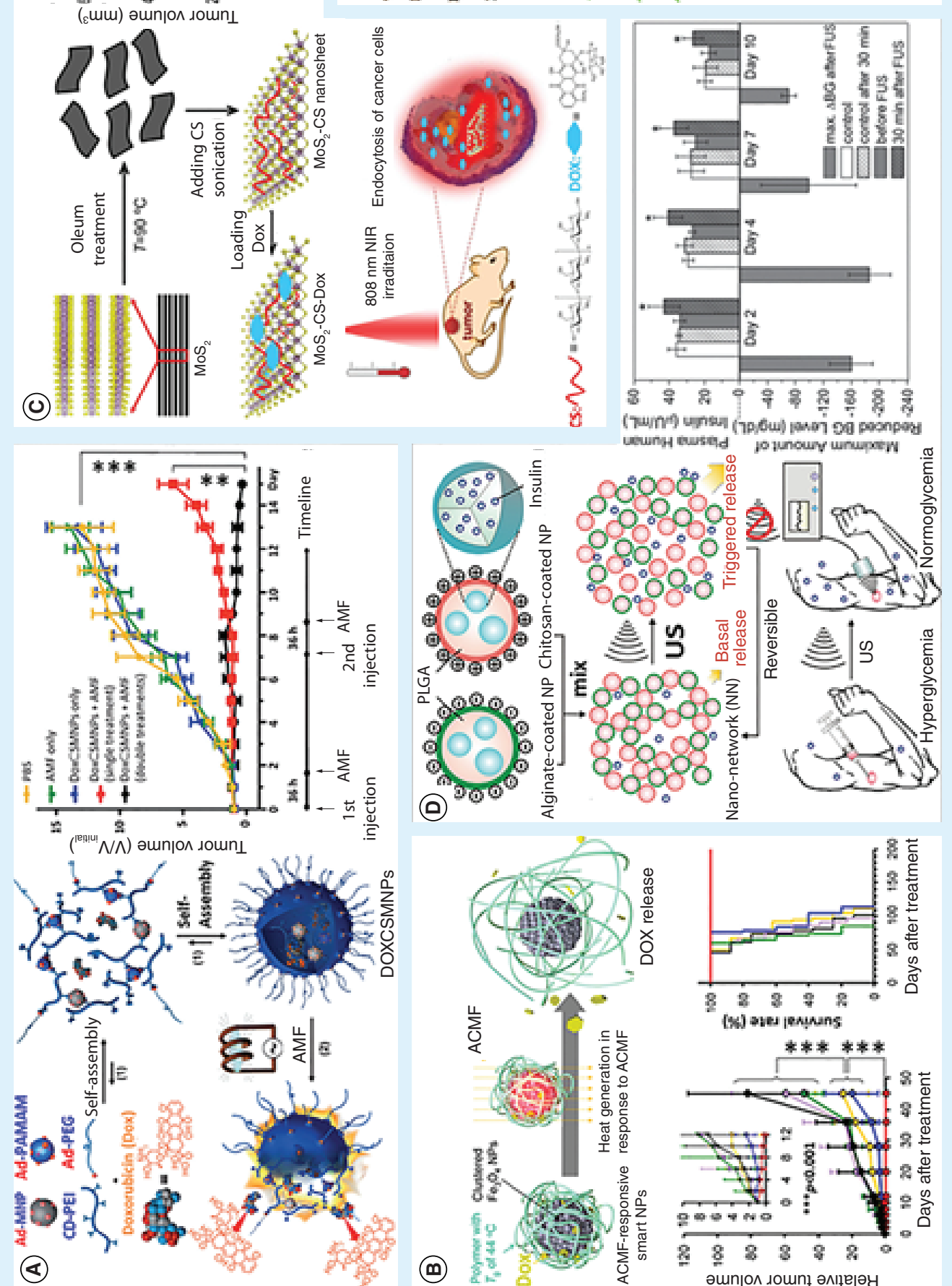

(0)

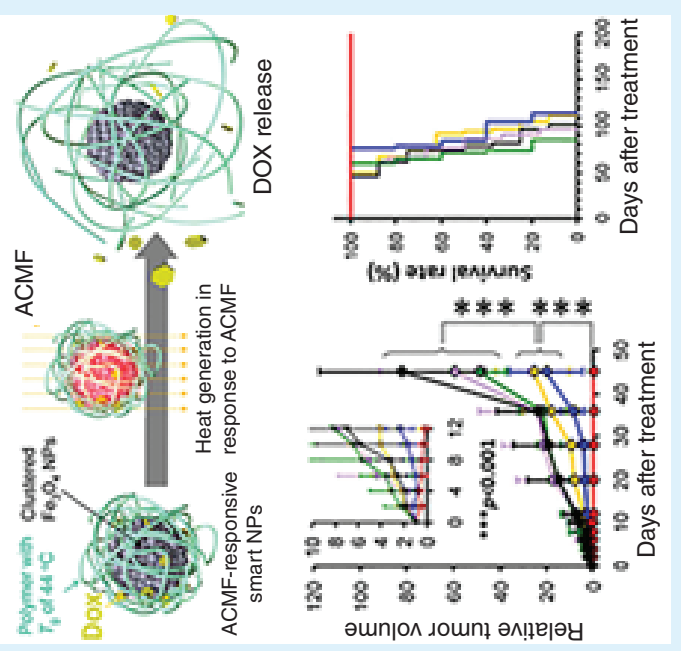

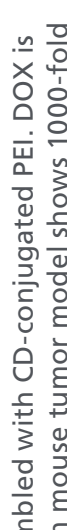

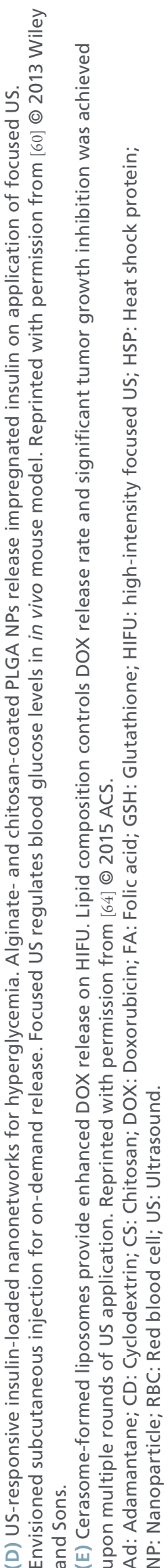


transition temperature $[\mathrm{Tg}]=44^{\circ} \mathrm{C}$ ) [48]. Decoration of the NPs with folic acid (FA) enabled specific targeting to FA receptor-expressing tumor cells in a mouse model of multiple myeloma. In response to an applied AMF, the polymer phase transitions leading to release of DOX (Figure 3B). This preparation showed excellent therapeutic efficacy in treating tumors in a single dose with complete remission and no significant toxicity noted [48]. Tietze et al. used superparamagnetic iron oxide NPs (SPIONs) as a carrier system for the delivery of the chemotherapeutic mitoxantrone to hind limb tumor implants in New Zealand white rabbits. An 80-fold improvement in drug accumulation within the tumor coupled with complete tumor remission was reported [49].

In a multi-NP approach, MNPs were combined with a photosensitizer (m-THPC; marketed as Foscan) within $200 \mathrm{~nm}$ liposomes [50]. The dual cargo liposomes could be stimulated via laser excitation to generate highly toxic reactive oxygen species (ROS) and by AMF to induce local hyperthermia. This coupling of photodynamic therapy with magnetic hyperthermia led to complete destruction of cells in vitro and complete eradication of epidermoid carcinoma-induced tumors in vivo. Cumulatively, these examples demonstrate the degree of flexibility and increasing design complexity that can be afforded by the magnetically actuated NP platform.

\section{Light-triggered actuation}

Light-induced stimulation is another attractive modality for active NP actuation as it facilitates a high degree of spatiotemporal control while enabling multiple routes for NP-cargo modulation including: the breaking of chemical bonds; generation of ROS; intitiation of molecular rearrangements/isomerization; and, thermal induction within and around the NP. Here, we discuss a number of recent examples that employ light as the NP actuation source.

The use of light for the induced cleavage of chemical bonds has become a powerful platform for the release of NP-associated cargos and has been facilitated by a number of photolabile linkers. Such linkers include $o$-nitrobenzyl, coumarin and quinolone. For example, $\mathrm{Hu}$ et al. used FA-functionalized dual-responsive shell cross-linked micelles that were covalently conjugated to $o$-nitrobenzyl photocaged camptothecin (CPT) prodrug [11]. Acylhydrazone and disulfide linkages in the micelle corona could be decross-linked by acidic and reductive cellular conditions, respectively. Upon photoactivation with UV light, the diffusion of CPT out of the micelle nanocarriers could be further modulated by external $\mathrm{pH}$ and thiol levels. The Almutairi group also used a light-responsive $o$-nitrobenzyl cross-linked polymer doped with the angiogenesis inhibitor nintedanib (BIBF 1120) and fluorescein (as a tracer for light-induced release). Nintedanib is a small molecule inhibitor of key receptors involved in new blood vessel formation in the eye. In a rat model of retinopathy the polymeric NPs retained the encapsulated drug and could 'on-demand' release the drug for up to 30 weeks post injection into the vitreous upon illumination with $365 \mathrm{~nm}$ light [12].

The previous examples utilize photolabile linkers that require high energy UV light that has limited tissue depth penetration and can potentially have off-target effects upon repeated stimulation. Accordingly, significant effort has been made to develop photo-triggered NP constructs that respond to lower energy, near infrared (NIR) excitation. Organic polymers and AuNPs that absorb in the NIR have attracted considerable interest. Qin et al., for example, synthesized chitosan-functionalized single-wall carbon nanotubes (CNT) that were encapsulated in a thermal/ $\mathrm{pH}$-responsive nanogel [51]. The chitosan facilitated the loading of DOX via $\pi-\pi$ stacking and NIR irradiation $(808 \mathrm{~nm})$ of the NPs induced CNT heating to promote on-demand DOX release. For long-term therapies, NPs that can realize repeated, on-demand, reproducible dosing would be a critically enabling technology. The Langer and Kohane groups developed a device containing a hydrophobic ethylcellulose matrix impregnated with hollow gold nanoshells loaded with aspart, a fast-acting insulin analog [52]. Over a 2-week period, controlled, on demand dosing was achieved in Sprague-Dawley rats using $808 \mathrm{~nm}$ NIR excitation. Hollow gold nanoshells were used in a similar fashion to deliver DOX to A549 cells [53]. Targeting was achieved by decoration of the NPs with an antiepidermal growth factor receptor antibody and DOX release was achieved by NIR irradiation. Interestingly, irradiation of DOX-NPs accumulated on the cell surface turned out to be more effective for cell killing compared with endocytosed DOX-NPs or free DOX. This suggested the important role of controlled release of DOX at the membrane and the avoidance of multidrug resistance efflux pumps. NIR excitation has also been used for the combinatorial photothermal drug delivery from $\mathrm{MoS}_{2}$ nanosheets. Yin et al. synthesized nanosheets of controlled size by an oleum treatment exfoliation process [54]. Decoration of the sheets with chitosan and loading with DOX followed by intratumoral injection and NIR excitation showed enhanced inhibition of tumor progression compared with free DOX or DOX-free irradiated sheets (Figure 3C).

Light has also been used to exploit the inherent physicochemical properties of NPs as a means to alter cellular function. In these examples, no cargo is 
released; the NP itself drives the modulation of cellular activity. For example, Lugo et al. showed the ability to use photo-excited QDs as an optical source probe to modulate the membrane potential of LnCap (prostate cancer) cells and cortical neurons [55]. Cells grown on $\mathrm{CdTe}$ and CdSe QD films were optically stimulated (via activation of voltage-gated ion channels) by illumination of membrane-proximal QDs. More recently, Carvalho-de-Souza and colleagues used membranetethered AuNPs illuminated with $532 \mathrm{~nm}$ light to drive thermal-induced changes in plasma membrane capacitance resulting in action potential generation in dorsal root ganglion cells [56]. This proof-of-concept study holds promise for NIR excitation stimulation given the variety of shapes of AuNPs possessing plasmon band absorption peaks ranging from $500 \mathrm{~nm}$ to $2 \mu \mathrm{m}$. The use of light-actuated NP materials for optoelectronic cellular stimulation presents the exciting possibility to use this modality in concert with current optogenetic approaches [57].

\section{Ultrasound-triggered actuation}

The application of ultrasonic (US) technology as a means of NP actuation offers some significant advantages over magnetic- or light-actuated systems. Most notably, US waves have superior transmission through a range of media (including biological tissue) which enables deeper tissue penetration and can simultaneously exert physical effects on both the tissue and the NP carrier. These effects include pressure variations (push and stress), acoustic fluid streaming, cavitation and local hyperthermia which collectively make US a unique external stimulus for NP-mediated drug delivery systems. In this section, we highlight a number of recent examples that employ US-actuated NP delivery. For further detail, the interested reader is directed to several excellent recent reviews [58,59].

Di et al. developed US-triggered PLGA NPs coated with alginate and chitosan for the on-demand delivery of insulin (Figure 3D) [60]. This delivery system displayed rapid and pulsatile regulation of blood glucose levels using a focused US source for up to 10 days after subcutaneous administration of the NPs. US-mediated actuation of therapeutic NPs has been used for the release of anticancer therapeutics from a range of NP materials. Yang et al., for example, generated nanocapsules based on disulfide cross-linked poly(methacrylic acid) (PMAA) for the controlled release of DOX [61]. This system was actually responsive to multiple stimuli (US, $\mathrm{pH}$ and reducing environment) to produce a three-in-one imaging, therapeutic and biodegradable drug delivery platform. Huebsch and coworkers developed a self-healing cross-linked hydrogel NP for the controlled US-induced delivery of mitoxantrone in a reversible 'on/off' manner [62]. Treatment of xenograft tumors was found to be most effective with a drug-laden gel combined with a daily US pulse. Ninomiya et al. employed US-triggered liposomes loaded with calcein and DOX and further comodified with single stranded DNA aptamers for recognition of platelet-derived growth factor receptors on breast cancer cells and poly(NIPMAM-co-NIPAM) as a thermosensitive polymer to sensitize the liposomes to high temperature. MDA-MB-231 cells were selectively killed by targeted US-actuation of the cell-bound liposomes and release of DOX while calcein provided confirmatory cell tracking [63]. Liang et al. demonstrated high intensity focused US (HIFU) with thermosensitive cerasome-formed liposomes (HTSCs) loaded with DOX [64]. The US-induced DOX release rate could be tightly controlled by titration of the lipid composition. Systemic (iv) administration of DOX-loaded HTSCs at $5 \mathrm{mg}$ DOX/kg coupled with double HIFU sonication significantly inhibited tumor growth of adenocarcinoma (MDA-MB-231) tumor-bearing mice (Figure 3E).

Finally, US-triggered NPs have been used for various gene delivery applications. Gao et al. developed phase-transition cationic nanodroplets based on perfluorinated amphiphilic poly(amino acid), which were simultaneously loaded with perfluoropentane and plasmid DNA [65]. This preparation showed a 14-fold enhancement of gene transfection efficiency on HepG2 cells upon US stimulation while affording good US contrast effect. Similarly, Wang and colleagues reported the delivery of microRNA-122 (miR122) encapsulated PEG-PLGA NPs in human colon cancer xenografts using US-induced cavitation [66]. Enhanced delivery of micro RNA-122 was achieved using optimal acoustic settings with minimal tissue damage. PEG-PLGA encapsulated miR-122 delivery into tumors with US and microbubbles was 7.9-fold higher compared with treatment without US.

\section{Active/intracellularly triggered NP actuation}

The active actuation of therapeutic NPs by intracellular stimuli relies on innate cellular processes or physiological states to achieve release of the drug cargo. Much of the work in this area has focused on three primary stimuli: $\mathrm{pH}$, enzymatic activity and redox potential and we focus on these here. Other stimuli (e.g., hypoxia, temperature) are also under development and for more comprehensive coverage the reader is directed to the excellent review by Wong and Choi [67].

\section{pH-triggered actuation}

The use of $\mathrm{pH}$ as the basis of controlled drug release from NPs stems from cancer biology where the low $\mathrm{pH}$ 
of the tumor environment [68] drives the release of the drug cargo from acid-responsive NP constructs. Examples include acid-labile, soft polymeric NPs or hard NPs (AuNPs, metal oxides) appended with drugs via acidresponsive surface linkages. Poly(2-(diisopropylamino) ethyl methacrylate (PDPA) was functionalized with zwitteronic polycarboxybetaine (PCB) methacrylate to prolong circulation time and with RGD peptides for tumor targeting. These constructs showed greater uptake of DOX in the tumor than other tissues in vivo [14]. Liang et al. formed multilayered PDPA capsules via layer-by-layer encapsulation that responded to low $\mathrm{pH}$ with interlayer repulsion causing release of drug cargos [69]. The Zare group reported on polypyrrole NPs for tunable $\mathrm{pH}$-sensitive drug release at both acidic (for positively charged drugs) and basic $\mathrm{pH}$ (for negatively charged drugs) by varying the charge of the drug and by addition of charged amphiphiles [70] (Figure 4A). Supramolecular peptide-amphiphile structures (SPAS) based on polypeptide self-assembly were shown to be responsive to acidic $\mathrm{pH}$ for the release of encapsulated drugs [71]. Poly- $L$-lysine and poly- $L$-leucine served as the hydrophilic and hydrophobic head and tail groups, respectively.

A number of 'hard' NP materials have been used for $\mathrm{pH}$-responsive drug delivery. Bhang et al. used the differential reduction potentials of manganese and gold to selectively promote release of $\mathrm{Mn}^{2+}$ ions from within AuNPs in the acidic environment of endosomes in PC12 pheochromocytoma cells [72]. This was used to enhance the neuro differentiation of these cells while decreasing apoptosis and necrosis. Zolata and coworkers recently modified In-111-labeled SPIONS with Trastuzumab-DOX attached by $\mathrm{pH}$-sensitive hydrazone bonds. This construct was used for combined tumor targeting, MRI and DOX release into the tumor environment [73].

\section{Enzyme-triggered actuation}

The release of drug cargos by intracellular enzymatic cleavage is another popular theme in active NP actuation. Liu et al. used mesoporous silica NPs (MSNs) for targeted tumor therapy in vitro and in vivo by capping MSNs with human serum albumin and decorating them with cell penetrating and matrix metalloproteinase 2 (MMP-2) substrate peptides [74]. The MMP-2 peptide carried attached DOX molecules that were cleaved and released by MMP-2 activity in HepG2 cells in vitro and in tumor-bearing mice in vivo (Figure 4B. Nitrogen-doped carbon nanotubes were used as nanocontainers corked with AuNPs to deliver paclitaxel to tumor-associated myeloid-derived suppressor cells (MDSC) via myeloperoxidase (MPO)-mediated release [75]. These nanoscale delivery containers simul- taneously delivered paclitaxel in an MPO-dependent fashion and blocked MDSC immunosuppressive potential (Figure 4C). Other enzyme-mediated actuation methods include supramolecular NPs comprised of sulfato- $\beta$-cyclodextrin and protamine that respond to trypsin digestion [76], aromatic azo-linkers that are cleaved specifically by azoreductase enzymes in hepatic cancer cells [77] and modified polystyrene that has multiple MMP-2 cleavable peptides [78].

\section{Redox-triggered actuation}

The reduction of disulfide bonds and dative sulfur interactions remains as the primary basis of redoxmediated intracellular cargo release from NPs. The high concentration of glutathione $(\sim 10 \mathrm{mM})$ in the cellular cytosol [79] provides strong reducing power for thiol-linked cargos and multiple recent examples point to the robustness of this approach. Wang et al., using a MSN platform, used dithiol-containing PEG chains to block/retain encapsulated drugs within the MSN core. The drug release rate was shown to correlate inversely with PEG grafting density [15]. Zhao and colleagues showed in MSNs the role played by thiol density in mediating drug carrying capacity. They used varying concentrations of the pendant attaching ligand, 3-mercaptopropyltrimethoxysilane (MPTMS), to determine the role of thiol group density on drug (6-mercaptopurine [6-MP]) loading efficiency and found that the presence of a high number thiol groups actually hindered drug loading due to steric hindrance in the MSN core [80] (Figure 4D). This group further showed the ability to use hyaluronic acid as a targeting ligand for CD44-mediated endocytosis of the 6-MP-loaded MSNs by HCT-116 cells [81]. Xu et al. used thiolated gelatin NPs loaded with plasmid encoding the $\mathrm{p} 53$ gene or conjugated with the antitumor drug gemcitabine for treatment of adenocarcinoma-bearing SCID mice. The drug- or plasmid-loaded gelatin NPs had better in vivo tumor suppression than either drug or plasmid alone [82] (Figure 4E. The intracellular redox environment has also been exploited to take advantage of the antioxidant properties of nanoceria for drug delivery. Muhammed et al. showed that $4 \mathrm{~nm}$ cerium oxide NPs camptothecin could be dissolved by intracellular vitamin $\mathrm{C}$ or glutathione to efficiently release the drug in BxPC-3 cells (pancreas adenocarcinoma) [83].

Several groups have created multifunctional NPs that are responsive to a number of the above intracellular stimuli. For targeted drug delivery to the colon, Naeem et al. used a combination of enzyme- and $\mathrm{pH}$ sensitive methyl methacrylate polymers to form NPs that were sensitive to both low $\mathrm{pH}$ in the stomach and small intestine as well as azoreductase produced by the microflora in the colon [84]. Together both triggers pro- 


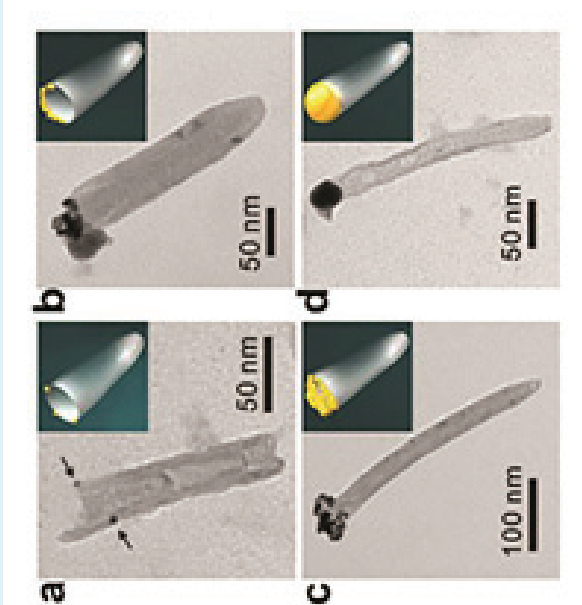

(2)

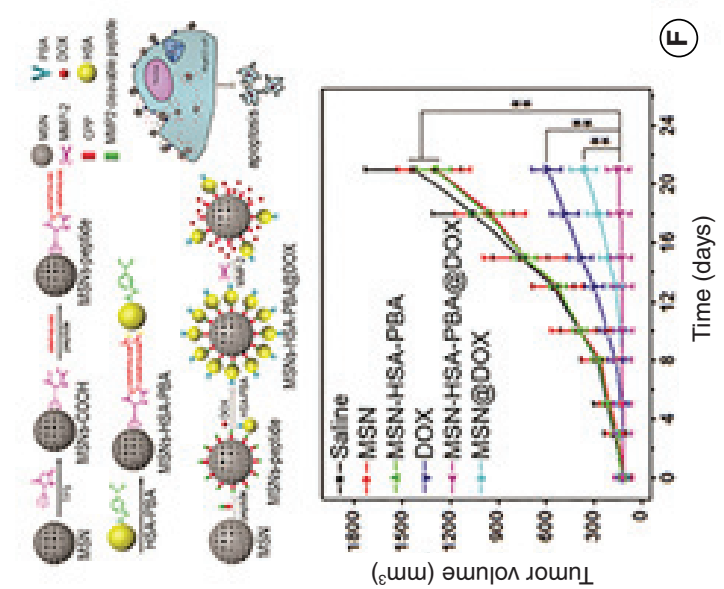

(a)

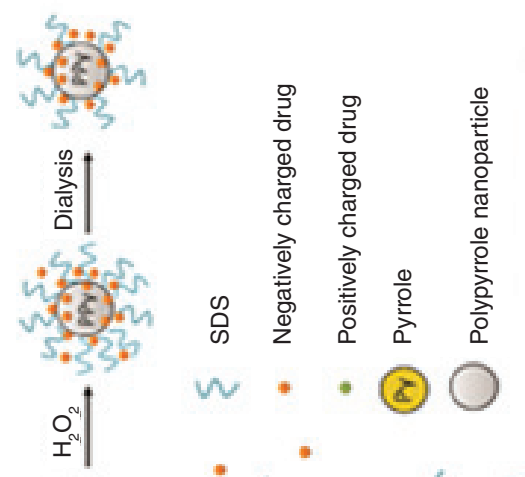

iscon

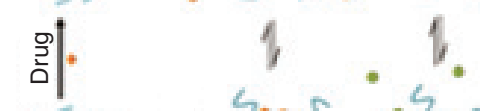

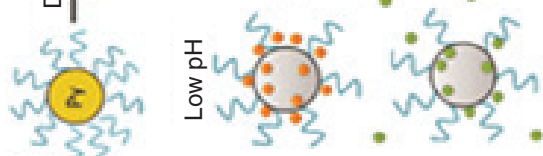

(4)

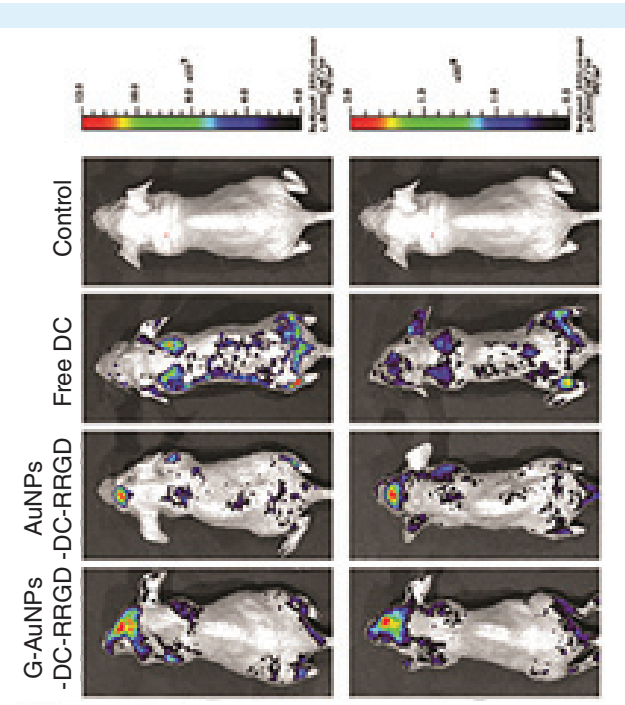

尔

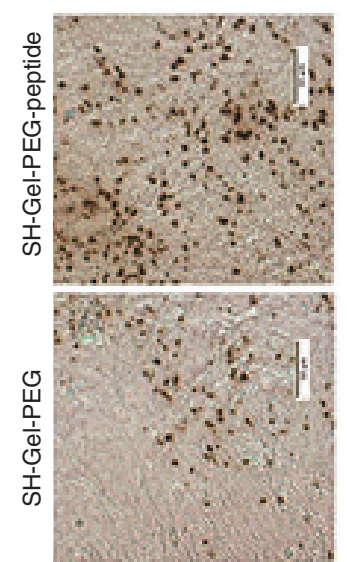

(4)
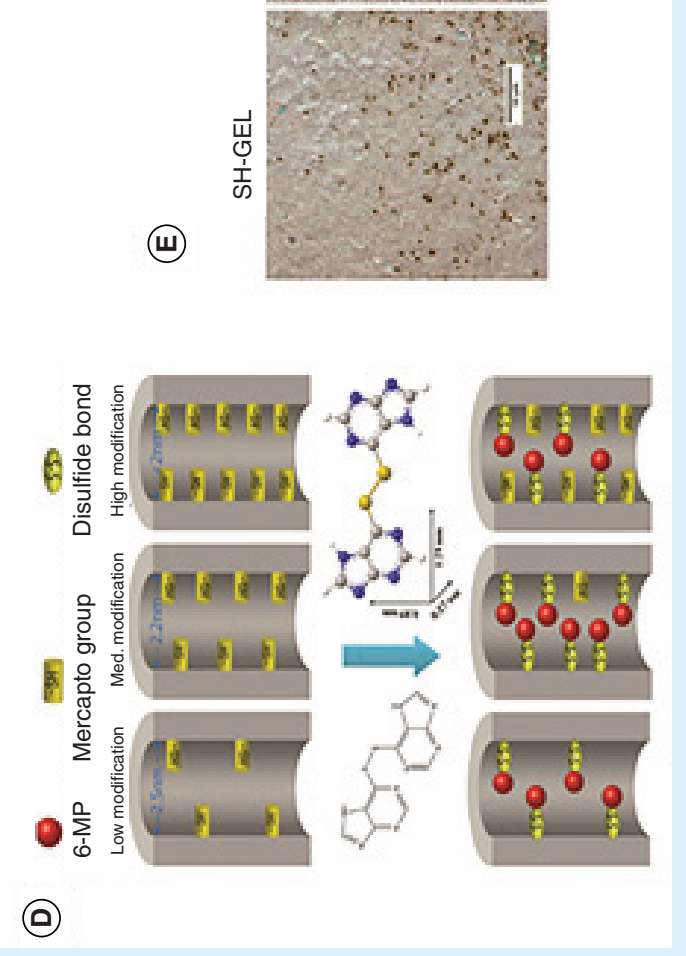

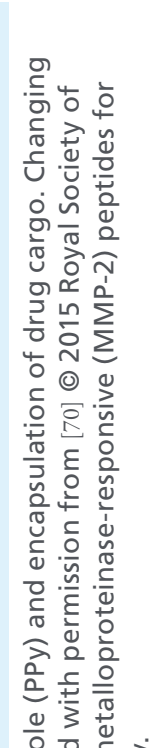

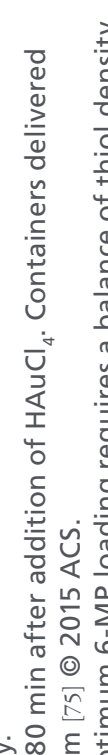

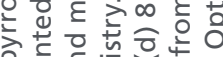

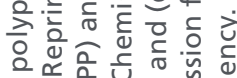

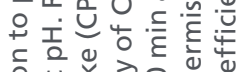

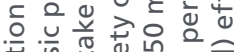

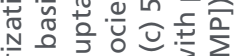

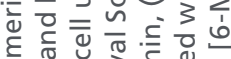

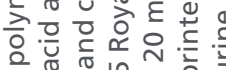

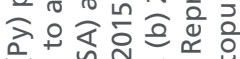

o

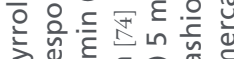

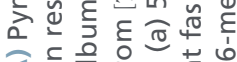

近

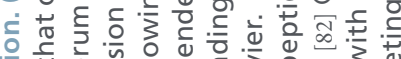

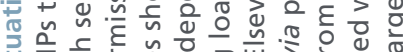

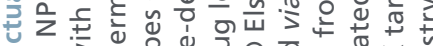

\%

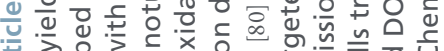

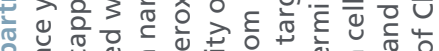

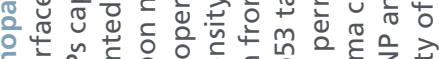

둔

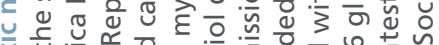

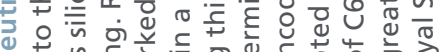

웜

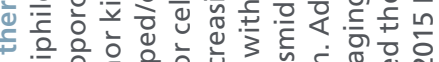

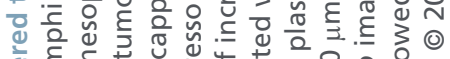

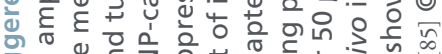

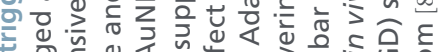
宊

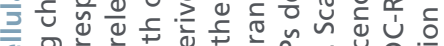
ष्षें

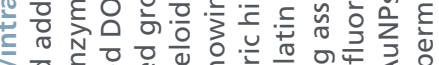

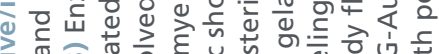

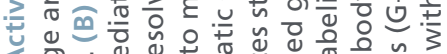

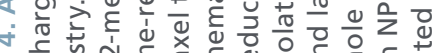

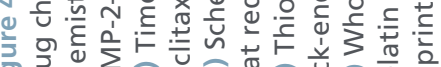

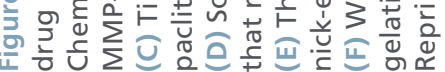


duced significant release of the drug in the colon and cecum compared with the stomach or small intestine in the rat model of colitis. Functionalization of gelatin NPs with a tumor-targeting peptide (RRGD) and AuNPs with acid-degradable linkers carrying DOX created a dual-triggered system that displayed preferential killing of glioma tumors in vivo [85] (Figure 4F). Gelatin is a natural MMP-2 substrate and gelatin digestion decreases NP size, allowing more efficient uptake of the drug-carrying AuNPs into the tumor. Cumulatively, these examples point to the elegant strategies that have been employed to achieve active/ intracellular-triggered NP-mediated drug delivery.

\section{Current clinical state of therapeutic NP actuation}

The ultimate application of the types of NP constructs described herein is in the clinical setting where the enhanced/modulated drug activity afforded by its delivery as an NP can translate into improved diagnosis, therapy and patient outcome. Candidate drugs for use in humans must gain approval by the US FDA's Center for Drug Evaluation and Research (CDER) through a structured evaluation process consisting of preclinical, clinical and postmarketing phases. The process is time-consuming, approval rates are low and those NP-drug formulations that have been approved to date (aimed at treating any number of cancers) represent some of the simplest NP constructs that utilize passive actuation as the drug delivery modality. Often, these constructs lack a targeting component; they simply rely on size-dependent, passive accumulation of the drug-loaded NP in the tumor tissue due to leaky tumor vasculature and the enhanced permeation and retention (EPR) effect. Examples here include the first approved nanodrug Doxil ${ }^{\circledR}$ (1995) which is a PEGylated liposomal form of DOX and more recently Abraxane (2005), which is an albumin NP form of the plant-derived taxane, paclitaxel. The latter is an excellent example of NP-enhanced solubility of an otherwise highly water-insoluble drug. The albumin carrier replaces previously employed, toxic surfactants to realize the increased bioavailability of paclitaxel and higher intratumor drug concentrations. More active NP targeting relies on the exploitation of overexpression of tumor cell markers. The carbohydrate antigen CA-125, for example, is expressed in more than $85 \%$ of ovarian cancers [86], yet active targeting NPs represent the vast minority of clinically approved NP-drug constructs. Of the dozen or so FDA-approved nanomedicines, nearly all of them utilize passive actuation coupled with EPR-mediated tumor accumulation. Likewise, the vast majority of currently running clinical trials involve NPs based on passive actuation; pacli- taxel either alone or in combination with another chemotherapeutic accounts for more than half of ongoing NP-drug clinical trials.

What does the current state of FDA-approved nanomedicines tell us? First, it is apparent that the simpler, passive NP actuation concepts have transitioned more rapidly into approved drugs than actively actuated NP-drug constructs. Clinical trials of actively actuated NPs are still progressing. The first clinical trial of NBTXR3 hafnium oxide nanocrystals that release free radicals upon irradiation of soft tissue sacrcoma recently completed (January 2016) and represents one of the first completed trials for active/extracellularly triggered actuation [87]. Similarly, the active intracellularly triggered CRLX101 (camptothecin-bound cyclodextrin polymer NPs) also recently completed its first clinical trial for treatment of adenocarcinoma [88]. We expect the number of clinical trials involving actively actuated NPs will continue to grow as our basic science understanding of these materials forges ahead.

\section{Future perspective}

In highlighting the work performed in the approximately 2 years period that encompasses this update review, it is clear that the complexity and sophistication of therapeutic NP constructs that are amenable to actuation are growing at an exponential rate. Looking ahead, what do we expect on the near- and long-term horizons? In the near-term, we expect to see more intricate NP designs/composites that incorporate multiple actuation modalities and incorporate multiple triggers. With that, comes a lot more basic research into how to assemble and control these constructs. The multicomponent $\mathrm{Au}$ and Ag NPs systems for combined magnetically assisted homing and thermally induced drug release described by Elbialy et al. are a good example of 'value-added' NP materials where multiple NP species yield an ensemble function not attainable by the constituent materials individually. Similarly, the work of Naeem et al. on multipolymer systems that are differentially responsive to $\mathrm{pH}$ and enzymatic degradation shows a pathway to NP systems that can potentially 'do more' in terms of responding simultaneously to multiple stimuli [84]. Further, as new methods for the isolation of novel and more specific tissue- and cell-type targeting ligands (including peptides and drug moieties) are realized [89], we can expect to see improved efficacy of therapeutic NP materials. Looking further down the road, we expect advancements in the pipeline of FDA-approved NP ensembles beyond the passive actuation constructs that are currently approved for use in the clinic. Clearly this will require the type of sustained investment in fundamental basic science that enables our understanding of how to actuate these 
materials at a high level with control and reproducibility. As these activities progress, so will our understanding of the short and long term toxicity profiles of NPs in the therapeutic setting and, more generally, in the environment [90-92].

\section{Financial \& competing interests disclosure}

The authors acknowledge the NRL Base Funding Program and the NRL Institute for Nanoscience for financial support. Okhil K Nag and YungChia Chen are supported by Research
Associateships through the NRC and ASEE, respectively. Lauren D Field and Ajmeeta Sangtani are PhD candidates in the Fischell Department of Bioengineering, University of Maryland College Park, MD. USA. The authors have no other relevant affiliations or financial involvement with any organization or entity with a financial interest in or financial conflict with the subject matter or materials discussed in the manuscript apart from those disclosed.

No writing assistance was utilized in the production of this manuscript.

\section{Executive summary}

\section{Use of NPs in therapeutic applications}

- The small size of nanoparticles (NPs) allows extended circulation times and deeper tissue penetration in vivo compared with other materials; significant surface area/volume allows loading of large 'payloads' on small platform.

- NPs for therapeutics can be either 'soft' (liposomes, micelles) or 'hard' (metal oxides, nanocrystals), offering surface and core loading or just surface loading, respectively.

- Significant progress continues to be made in the 'actuation' of NP-cargo conjugates.

NP actuation modalities

- Passive, active/extracellularly triggered, active/intracellularly triggered are the major modalities NP cargo actuation.

- Passive actuation involves simple efflux of cargo from NP; active modalities utilize various triggers: magnetic, light, ultrasonic, $\mathrm{pH}$, redox and enzyme-mediated.

Integrating NP actuation into cells \& tissues

- Therapeutic uses of NP-cargo constructs is a balance of nonspecific binding, circulation time, targeting, cell/ tissue uptake and clearance.

- The optimum mode of NP-based delivery and coupled actuation modality is dependent on the toxicity of the free drug.

Clinical state of NP actuation for therapeutics

- Simple, yet elegant, passive NP actuation designs (e.g., desorption, efflux) remain the only US FDA-approved modality for use in the clinic.

- Basic research demonstrations of actively actuated, multicomponent NPs suggests these constructs will soon be tested in clinical trials.

\section{References}

Papers of special note have been highlighted as:

- of interest $\bullet$ of considerable interest

1 Delehanty JB, Mattoussi H, Medintz IL. Delivering quantum dots into cells: strategies, progress and remaining issues. Anal. Bioanal. Chem. 393(4), 1091-1105 (2009).

2 Delehanty JB, Susumu K, Manthe RL, Algar WR, Medintz IL. Active cellular sensing with quantum dots: transitioning from research tool to reality; a review. Anal. Chim. Acta 750, 63-81 (2012).

3 Ruedas-Rama MJ, Walters JD, Orte A, Hall EA. Fluorescent nanoparticles for intracellular sensing: a review. Anal. Chim. Acta 751, 1-23 (2012).

4 Roldo M. Carbon nanotubes in drug delivery: just a carrier? Ther. Deliv. 7(2), 55-57 (2016).

5 Nazarenus M, Zhang Q, Soliman MG et al. In vitro interaction of colloidal nanoparticles with mammalian cells: what have we learned thus far? Beilstein. J. Nanotechnol. 5 , 1477-1490 (2014).
- An excellent and timely review on the currently accepted concepts regarding the in vitro interaction of colloidal nanoparticles with mammalian cells.

6 Paranjpe M, Müller-Goymann C. Nanoparticle-mediated pulmonary drug delivery: a review. Int. J. Mol. Sci. 15(4), 5852 (2014).

7 Ryvolova M, Chomoucka J, Drbohlavova J et al. Modern micro and nanoparticle-based imaging techniques. Sensors, 12(11), 14792 (2012).

8 Patel SC, Lee S, Lalwani G, Suhrland C, Chowdhury SM, Sitharaman B. Graphene-based platforms for cancer therapeutics. Ther. Deliv. 7(2), 101-116 (2016).

9 Delehanty JB, Breger JC, Gemmill KB, Stewart MH, Medintz IL. Controlling the actuation of therapeutic nanomaterials: enabling nanoparticle-mediated drug delivery. Ther. Deliv. 4(11), 1411-1429 (2013).

-. A comprehensive review (through 2013) covering the various passive and active actuation modalities for mediating the controlled release of therapeutic cargos from nanoparticle carriers. 
10 Delehanty JB, Medintz IL. Controlled actuation of therapeutic nanoparticles: moving beyond passive delivery modalities. Ther. Deliv. 4(2), 127-129 (2013).

11 Hu X, Tian J, Liu T, Zhang G, Liu S. Photo-triggered release of caged camptothecin prodrugs from dually responsive shell cross-linked micelles. Macromolecules 46(15), 6243-6256 (2013).

12 Huu VAN, Luo J, Zhu J et al. Light-responsive nanoparticle depot to control release of a small molecule angiogenesis inhibitor in the posterior segment of the eye. J. Control. Rel. 200(0), 71-77 (2015).

13 Lee J-H, Chen K-J, Noh S-H et al. On-demand drug release system for in vivo cancer rreatment through self-assembled magnetic nanoparticles. Angew Chem. Int. Ed. Engl. 52(16), 4384-4388 (2013).

- A highly effective demonstration of magnetothermally responsive drug-encapsulated supramolecular nanoparticles for on-demand drug release and treatment of cancer in vivo.

14 Huang P, Song H, Wang W et al. Integrin-rargeted zwitterionic polymeric nanoparticles with acid-induced disassembly property for enhanced drug accumulation and release in tumor. Biomacromolecules 15(8), 3128-3138 (2014).

15 Wang Y, Han N, Zhao Q et al. Redox-responsive mesoporous silica as carriers for controlled drug delivery: a comparative study based on silica and PEG gatekeepers. Eur. J. Pharm. Sci. 72, 12-20 (2015).

16 Matsumura Y, Yoshikata K, Kunisaki S, Tsuchido T. Mode of bactericidal action of silver zeolite and its comparison with that of silver nitrate. Appl. Environ. Microb. 69(7), 4278-4281 (2003).

17 Chen X, Schluesener HJ. Nanosilver: a nanoproduct in medical application. Toxicol. Lett. 176(1), 1-12 (2008).

18 Prabhu S, Poulose E. Silver nanoparticles: mechanism of antimicrobial action, synthesis, medical applications, and toxicity effects. Int. Nano Lett. 2(1), 1-10 (2012).

19 Kasivelu Govindaraju KK, Alsagaby SA, Ganesan SMP. Green synthesis of silver nanoparticles for selective toxicity towards cancer cells. IET Nanobiotechnol. 9(6), 325-330 (2015).

20 Suliman YA, Ali D, Alarifi S, Harrath AH, Mansour L, Alwasel SH. Evaluation of cytotoxic, oxidative stress, proinflammatory and genotoxic effect of silver nanoparticles in human lung epithelial cells. Environ. Toxicol. 30(2), 149-160 (2015).

21 Zhang $\mathrm{H}$, Wang $\mathrm{X}$, Wang $\mathrm{M}$ et al. Mammalian cells exhibit a range of sensitivities to silver nanoparticles that are partially explicable by variations in antioxidant defense and metallothionein expression. Small 11(31), 3797-3805 (2015).

22 Oh E, Delehanty JB, Sapsford KE et al. Cellular uptake and fate of PEGylated gold nanoparticles is dependent on both cell-penetration peptides and particle size. ACS Nano. 5(8), 6434-6448 (2011).

- A detailed study on the size-dependent nature of cellular internalization of gold nanoparticles mediated by polyarginine cell-penetrating peptides.
23 Alkilany AM, Murphy CJ. Toxicity and cellular uptake of gold nanoparticles: what we have learned so far? J. Nanopart. Res. 12(7), 2313-2333 (2010).

24 Seo JM, Kim EB, Hyun MS, Kim BB, Park TJ. Self-assembly of biogenic gold nanoparticles and their use to enhance drug delivery into cells. Colloids Surf. B Biointerfaces 135, 27-34 (2015).

25 Elbialy NS, Fathy MM, Khalil WM. Doxorubicin loaded magnetic gold nanoparticles for in vivo targeted drug delivery. Int. J. Pharm. 490(1-2), 190-199 (2015).

26 Afifi MM, Austin LA, Mackey MA, El-Sayed MA. XAV939: from a small inhibitor to a potent drug bioconjugate when delivered by gold nanoparticles. Bioconjug. Chem. 25(2), 207-215 (2014).

27 Mu Q, Kievit FM, Kant RJ, Lin G, Jeon M, Zhang M. Anti-HER2/neu peptide-conjugated iron oxide nanoparticles for targeted delivery of paclitaxel to breast cancer cells. Nanoscale, 7(43), 18010-18014 (2015).

28 Hsiao M-H, Mu Q, Stephen ZR, Fang C, Zhang M. Hexanoyl-Chitosan-PEG Copolymer Coated Iron Oxide Nanoparticles for Hydrophobic Drug Delivery. ACS Macro. Lett. 4(4), 403-407 (2015).

29 Kossatz S, Grandke J, Couleaud P et al. Efficient treatment of breast cancer xenografts with multifunctionalized iron oxide nanoparticles combining magnetic hyperthermia and anti-cancer drug delivery. Breast Cancer Res. 17, 66 (2015).

30 Chen X, Tang Y, Cai B, Fan H. 'One-pot' synthesis of multifunctional GSH-CdTe quantum dots for targeted drug delivery. Nanotechnology 25(23), 235101 (2014).

31 Wang X, Sun X, Lao J et al. Multifunctional graphene quantum dots for simultaneous targeted cellular imaging and drug delivery. Colloids Surf. B Biointerfaces 122, 638-644 (2014).

32 Murata M, Narahara S, Kawano T et al. Design and function of engineered protein nanocages as a drug delivery system for targeting pancreatic cancer cells via neuropilin-1. Mol. Pharm. 12(5), 1422-1430 (2015).

33 Bellini M, Mazzucchelli S, Galbiati E et al. Protein nanocages for self-triggered nuclear delivery of DNAtargeted chemotherapeutics in Cancer Cells. J. Control. Rel. 196, 184-196 (2014).

- A detailed study of the use of ferritin protein nanocages for the enhanced nuclear delivery and efficacy of doxorubicin.

34 Kambhampati SP, Mishra MK, Mastorakos P, Oh Y, Lutty GA, Kannan RM. Intracellular delivery of dendrimer triamcinolone acetonide conjugates into microglial and human retinal pigment epithelial cells. Eur. J. Pharm. Biopharm. 95(Pt B), 239-249 (2015).

35 Scarano W, de Souza P, Stenzel MH. Dual-drug delivery of curcumin and platinum drugs in polymeric micelles enhances the synergistic effects: a double act for the treatment of multidrug-resistant cancer. Biomater. Sci. 3(1), 163-174 (2015).

36 Kroon J, Buijs JT, van der Horst G et al. Liposomal delivery of dexamethasone attenuates prostate cancer bone metastatic tumor growth in vivo. Prostate 75(8), 815-824 (2015). 
Firneiro ZA, Mascharak P, Curti C, da Silva RS. Incorporation of a ruthenium nitrosyl complex into liposomes, the nitric oxide released from these liposomes and HepG2 cell death mechanism. Coord. Chem. Rev. 306, 701-707 (2016).

38 Ding Q, Si X, Liu D et al. Targeting and liposomal drug delivery to CD40L expressing T cells for treatment of autoimmune diseases. J. Control. Rel. 207, 86-92 (2015). drug delivery enabled by stable peptide ligands. J. Control. Rel. 218, 13-21 (2015). target-specific liposomes for delivering small molecule drugs after reperfused myocardial infarction. J. Control. Rel. 220(Pt A), 556-567 (2015).

41 Moles E, Urban P, Jimenez-Diaz MB et al. Immunoliposomemediated drug delivery to Plasmodium-infected and noninfected red blood cells as a dual therapeutic/prophylactic antimalarial strategy. J. Control. Rel. 210, 217-229 (2015).

42 Spillmann CM, Naciri J, Algar WR, Medintz IL, Delehanty JB. Multifunctional liquid crystal nanoparticles for intracellular fluorescent imaging and drug delivery. ACS Nano. 8(7), 6986-6997 (2014).

43 Wang C-F, Mäkilä EM, Kaasalainen MH et al. Dual-drug delivery by porous silicon nanoparticles for improved cellular uptake, sustained release, and combination therapy. Acta Biomater. 16, 206-214 (2015).

44 Khandelia R, Bhandari S, Pan UN, Ghosh SS, Chattopadhyay A. Gold nanocluster embedded albumin nanoparticles for two-photon imaging of cancer cells accompanying drug delivery. Small 11(33), 4075-4081 (2015).

45 Nigam P, Waghmode S, Louis M, Wangnoo S, Chavan P, Sarkar D. Graphene quantum dots conjugated albumin nanoparticles for targeted drug delivery and imaging of pancreatic cancer. J. Mater. Chem. B 2(21), 3190-3195 (2014).

46 Park E-J, Umh H, Choi D-H et al. Magnetite- and maghemite-induced different toxicity in murine alveolar macrophage cells. Arch. Toxicol. 88(8), 1607-1618 (2014).

47 Soenen SJ, De Cuyper M, De Smedt SC, Braeckmans $K$. Chapter ten - investigating the toxic effects of iron oxide nanoparticles. In: Methods Enzymol. Nejat D (Ed.). Academic Press 195-224 (2012).

Hayashi K, Nakamura M, Miki H et al. Magnetically responsive smart nanoparticles for cancer treatment with a combination of magnetic hyperthermia and remote-control drug release. Theranostics 4(8), 834-844 (2014).

49 Tietze R, Lyer S, Dürr $S$ et al. Efficient drug-delivery using magnetic nanoparticles - biodistribution and therapeutic effects in tumour bearing rabbits. Nanomedicine $9(7)$, 961-971 (2013).

50 Di Corato R, Béalle G, Kolosnjaj-Tabi J et al. Combining magnetic hyperthermia and photodynamic therapy for tumor ablation with photoresponsive magnetic liposomes. ACS Nano. 9(3), 2904-2916 (2015).
51 Qin Y, Chen J, Bi Y et al. Near-infrared light remotecontrolled intracellular anti-cancer drug delivery using thermo/pH sensitive nanovehicle. Acta Biomater.17(0), 201-209 (2015).

52 Timko BP, Arruebo M, Shankarappa SA et al. Nearinfrared-actuated devices for remotely controlled drug delivery. Proc. Natl Acad. Sci. USA 111(4), 1349-1354 (2014).

53 Noh MS, Lee S, Kang H et al. Target-specific near-IR induced drug release and photothermal therapy with accumulated $\mathrm{Au} / \mathrm{Ag}$ hollow nanoshells on pulmonary cancer cell membranes. Biomaterials 45(0), 81-92 (2015).

54 Yin W, Yan L, Yu J et al. High-throughput synthesis of single-layer MoS2 nanosheets as a near-infrared photothermal-triggered drug delivery for effective eancer therapy. ACS Nano. 8(7), 6922-6933 (2014).

55 Lugo K, Miao X, Rieke F, Lin LY. Remote switching of cellular activity and cell signaling using light in conjunction with quantum dots. Biomed. Opt. Express 3(3), 447-454 (2012).

56 Carvalho-de-Souza João L, Treger Jeremy S, Dang B, Kent Stephen BH, Pepperberg David R, Bezanilla F. Photosensitivity of neurons enabled by cell-targeted gold nanoparticles. Neuron 86(1), 207-217 (2015).

57 Packer AM, Roska B, Hausser M. Targeting neurons and photons for optogenetics. Nat. Neurosci. 16(7), 805-815 (2013).

58 Husseini GA, Pitt WG, Martins AM. Ultrasonically triggered drug delivery: breaking the barrier. Colloids Surf. B Biointerface 123, 364-386 (2014).

59 Sirsi SR, Borden MA. State-of-the-art materials for ultrasound-triggered drug delivery. Adv. Drug Deliv. Rev. 72, 3-14 (2014).

- A comphrehensive review of materials and mechanistic aspects of ultrasound-triggerable NP/cargo complex for controlled cargo release.

60 Di J, Price J, Gu X, Jiang X, Jing Y, Gu Z. Ultrasoundtriggered regulation of blood glucose levels using injectable nano-network. Adv. Healthc. Mater. 3(6), 811-816 (2014).

- An excellent study demonstrated on-demand release of insulin from subcutaneously injected nano-network using ultrasound.

61 Yang P, Li D, Jin S et al. Stimuli-responsive biodegradable poly(methacrylic acid) based nanocapsules for ultrasound traced and triggered drug delivery system. Biomaterials 35(6), 2079-2088 (2014).

62 Huebsch N, Kearney CJ, Zhao X et al. Ultrasoundtriggered disruption and self-healing of reversibly cross-linked hydrogels for drug delivery and enhanced chemotherapy. Proc. Natl Acad Sci. USA 111(27), 97629767 (2014).

63 Ninomiya K, Yamashita T, Kawabata S, Shimizu N. Targeted and ultrasound-triggered drug delivery using liposomes co-modified with cancer cell-targeting aptamers and a thermosensitive polymer. Ultrason. Sonochem. 21(4), 1482-1488 (2014). 
64 Liang X, Gao J, Jiang L et al. Nanohybrid liposomal cerasomes with good physiological stability and rapid temperature responsiveness for high intensity focused ultrasound triggered local chemotherapy of cancer. ACS Nano. 9(2), 1280-1293 (2015).

65 Gao D, Xu M, Cao Z et al. Ultrasound-triggered phasetransition cationic nanodroplets for enhanced gene delivery. ACS Appl. Mater. Interfaces 7(24), 13524-13537 (2015).

66 Wang T-Y, Choe JW, Pu K et al. Ultrasound-guided delivery of microRNA loaded nanoparticles into cancer. J. Control. Rel. 203, 99-108 (2015).

67 Wong PT, Choi SK. Mechanisms of drug release in nanotherapeutic delivery systems. Chem. Rev. 115(9), 3388-3432 (2015).

-• An excellent review on the controlled release of cargos in nanoparticle-mediated drug delivery systems. This review paticularly highlights the important chemistries one needs to consider.

68 Kato Y, Ozawa S, Miyamoto C et al. Acidic extracellular microenvironment and cancer. Cancer Cell. Int. 13(1), 1-8 (2013).

69 Liang K, Such GK, Johnston AP et al. Endocytic pHtriggered degradation of nanoengineered multilayer capsules. Adv. Mater. 26(12), 1901-1905 (2014).

70 Samanta D, Meiser JL, Zare RN. Polypyrrole nanoparticles for tunable, $\mathrm{pH}$-sensitive and sustained drug release. Nanoscale 7(21), 9497-9504 (2015).

$71 \mathrm{Xu}$ X, Li Y, Li H et al. Smart nanovehicles based on $\mathrm{pH}-$ triggered disassembly of supramolecular peptide-amphiphiles for efficient intracellular drug delivery. Small 10(6), 1133-1140 (2014).

72 Bhang SH, Han J, Jang HK et al. pH-triggered release of manganese from MnAu nanoparticles that enables cellular neuronal differentiation without cellular toxicity. Biomaterials 55, 33-43 (2015).

73 Zolata H, Abbasi Davani F, Afarideh H. Synthesis characterization and theranostic evaluation of Indium-111 labeled multifunctional superparamagnetic iron oxide nanoparticles. Nucl. Med. Biol. 42(2), 164-170 (2015).

74 Liu J, Zhang B, Luo Z et al. Enzyme responsive mesoporous silica nanoparticles for targeted tumor therapy in vitro and in vivo. Nanoscale 7(8), 3614-3626 (2015).

75 Zhao Y, Burkert SC, Tang Y et al. Nano-gold corking and enzymatic uncorking of carbon nanotube cups. J. Am. Chem. Soc. 137(2), 675-684 (2015).

76 Hou XF, Chen Y, Liu Y. Enzyme-responsive protein/ polysaccharide supramolecular nanoparticles. Soft Matter. 11(12), 2488-2493 (2015).

77 Medina SH, Chevliakov MV, Tiruchinapally G, Durmaz YY, Kuruvilla SP, Elsayed ME. Enzyme-activated nanoconjugates for tunable release of doxorubicin in hepatic cancer cells. Biomaterials 34(19), 4655-4666 (2013).

78 Guarnieri D, Biondi M, Yu H et al. Tumor-activated prodrug (TAP)-conjugated nanoparticles with cleavable domains for safe doxorubicin delivery. Biotechnol. Bioeng. 112(3),
601-611 (2015).

79 Hwang C, Sinskey A, Lodish H. Oxidized redox state of glutathione in the endoplasmic reticulum. Science 257(5076), 1496-1502 (1992).

80 Zhao Q, Wang C, Liu Y et al. PEGylated mesoporous silica as a redox-responsive drug delivery system for loading thiolcontaining drugs. Int. J. Pharm. 477(1-2), 613-622 (2014).

81 Zhao Q, Geng H, Wang Y et al. Hyaluronic acid oligosaccharide modified redox-responsive mesoporous silica nanoparticles for targeted drug delivery. ACS Appl. Mater. Interfaces6(22), 20290-20299 (2014).

$82 \mathrm{XuJ}$, Singh A, Amiji MM. Redox-responsive targeted gelatin nanoparticles for delivery of combination wt-p 53 expressing plasmid DNA and gemcitabine in the treatment of pancreatic cancer. BMC Cancer 14, 75 (2014).

83 Muhammad F, Wang A, Qi W, Zhang S, Zhu G. Intracellular antioxidants dissolve man-made antioxidant nanoparticles: using redox vulnerability of nanoceria to develop a responsive drug delivery system. ACS Appl. Mater. Interfaces 6(21), 19424-19433 (2014).

84 Naeem M, Kim W, Cao J, Jung Y, Yoo JW. Enzyme/pH dual sensitive polymeric nanoparticles for targeted drug delivery to the inflamed colon. Colloids Surf. B Biointerfaces 123, 271-278 (2014).

85 Ruan S, He Q, Gao H. Matrix metalloproteinase triggered size-shrinkable gelatin-gold fabricated nanoparticles for tumor microenvironment sensitive penetration and diagnosis of glioma. Nanoscale7(21), 9487-9496 (2015).

86 Moore RG, Brown AK, Miller MC et al. The use of multiple novel tumor biomarkers for the detection of ovarian carcinoma in patients with a pelvic mass. Gynecol Oncol.108(2), 402-408 (2008).

87 NBTXR3 crystalline nanoparticles and radiation therapy in treating patients with soft tissue sarcoma of the extremity. clinicaltrials.gov/show/NCT01433068

88 Pilot trial of crlx101 in treatment of patients with advanced or metastatic stomach, gastroesophageal, or esophageal cancer that cannot be removed by surgery. clinicaltrials.gov/ show/NCT01612546

89 Gao S, Simon MJ, Hue CD, Morrison B, 3rd, Banta S. An unusual cell penetrating peptide identified using a plasmid display-based functional selection platform. ACS Chem. Biol.6(5), 484-491 (2011).

90 Sajid M, Ilyas M, Basheer C et al. Impact of nanoparticles on human and environment: review of toxicity factors, exposures, control strategies, and future prospects. Environ. Sci. Pollut. Res. Int.22(6), 4122-4143 (2014).

91 Liu B, Campo EM, Bossing T. Drosophila embryos as model to assess cellular and developmental toxicity of multi-walled carbon nanotubes (MWCNT) in living organisms. PLoS One9(2), e88681 (2014).

92 Arami H, Khandhar A, Liggitt D, Krishnan KM. In vivo delivery, pharmacokinetics, biodistribution and toxicity of iron oxide nanoparticles. Chem. Soc. Rev.44(23), 8576-8607 (2015). 